\title{
On a new type of fractional difference operators on h-step isolated time scales
}

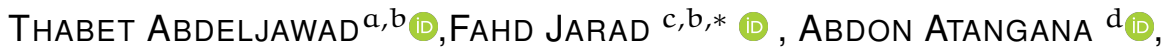 \\ PShtiwan Othman Mohammed ${ }^{e}{ }^{\infty}$ \\ ${ }^{a}$ Department of Mathematics and General Sciences, Prince Sultan University \\ P. O. Box 66833, 11586 Riyadh, Saudi Arabia \\ b Department of Medical Research, China Medical University, Taichung 40402, Taiwan \\ c Department of Mathematics, Çankaya University, Ankara 06790, Turkey \\ d Institute for Groundwater Studies, Faculty of Natural and Agricultural Sciences, University \\ of the Free State, 9301, Bloemfontein, South Africa \\ e Department of Mathematics, College of Education, University of Sulaimani, Sulaimani, \\ Kurdistan Region, Iraq
}

- Received: 17.02.2021 • Accepted: 02.03.2021 • Published Online: 06.03.2021

\begin{abstract}
In this article, a new type of fractional sums and differences called the discrete weighted fractional operators are presented. The weighted backward and forward difference operators are defined on an isolated time scale with arbitrary step size and they obey the power law.

Keywords: weighted fractional sum, weighted fractional difference, Caputo fractional weighted difference, Q-operator, Leibniz rule, isolated time scale, integration by parts, nabla discrete Mittag-Leffler function.

2010 MSC: 34A08, 26A33, 39A12.
\end{abstract}

\section{Introduction}

The continuous fractional calculus is a rich field of research and it has attracted the attention of several researchers working in diverse fields of science, engineering, economics and medicine [1, 2]. In order to meet the requirements of researchers dealing with models, it becomes essential to present new fractional operators with different kernels and weights. Very recently, the authors in [3] presented new generalized fractional operators with kernel depending on a function and by using a weight function acting

\footnotetext{
*Corresponding author: fahd@cankaya.edu.tr
}

(c) 2020 SABA. All Rights Reserved. 
inside and outside the integral operator used in the definition. Moreover, they studied many basic properties that are essential for researchers to apply theoretically and use in their modelling problems.

On the flipside, the discrete counterpart of the continuous fractional calculus has been playing an important role in meeting the modelling of many discrete emerging problems $[4,5,6,7,8,9,10,11,12,13,14]$.

Motivated by these, in this article and by the help of the time scale theory [15, 16], we present a detailed study that set the main concepts of the weighted discrete fractional calculus on the $h \mathbb{Z}$ time scale in the setting of nabla and delta differences for both left and right cases.

Our manuscript is organized as follow. In Section 2 we give the basic terminology and tools divided into three subsections (the rising and falling functions, Mittag-Leffler functions on $h \mathbb{Z}$ within nabla, the delta and nabla fractional sums and differences on $h \mathbb{Z}$, where the action of the Q-operator has been stated ), in Section 3, the left and right weighted fractional sums and differences into delta and nabla are proposed with some basic essential properties. In Section 4, left and right nabla and delta weighted Caputo fractional differences are defined and related to the weighted Rieman-Liouville sense ones. In Section 5, the Leibniz type rules showing the action of the weighted fractional sums on the Caputo fractional sums are discussed into nabla and delta and an initial value weighted difference problem is proposed in the Riemann-Liouville case. In Section 6, the integration by parts allowing the interchange between the weighted Riemann-Liouville and and Caputo fractional differences from left to right are proposed in both the nabla and delta cases. In Section 7, some examples are given in the nabla sense, where the solution of the linear nonhomogeneous equation is given explicitly by using a transformation method via the non-weighted case and expressed by means of the nabla discrete Mittag-Leffler function on the isolated time scale. Finally, we summarize our conclusions in Section 8.

\section{Preliminary tools and definitions}

This section contains the basic essential terms and tools that will be vital to proceed in the main results. Mainly, it sets the basic concepts on the time scale $h \mathbb{Z}[16]$ in the nabla sense. We borrow most of the notations from [9, 11, 12].

\subsection{The rising and falling factorials and nabla discrete Mittag-Leffler functions}

This subsection includes the basic concepts that are necessary to define the kernels of the nabla and delta fractional sums and differences. In addition, it contains the definition of the the nabla discrete Mittag-Leffler function that will be used in solving nabla linear fractional difference equations.

Definition 2.1. [5] The following identities are valid.

(i) Let $i$ be a natural number, then the $i$ rising factorial of $\tau$ is written as

$$
\tau^{\bar{i}}=\prod_{k=0}^{i-1}(\tau+k), \quad \tau^{\overline{0}}=1 .
$$


(ii) For any real number, the $\alpha$ rising function becomes

$$
\tau^{\bar{\alpha}}=\frac{\Gamma(+\alpha)}{\Gamma(\mathrm{t})}, \quad \text { such that } \tau \in \mathbb{C} \backslash\{\ldots,-2,-1,0\}, 0^{\bar{\alpha}}=0 .
$$

In addition, we have

$$
\nabla\left(\tau^{\bar{\alpha}}\right)=\alpha \tau^{\overline{\alpha-1}}
$$

Hence $\tau^{\bar{\alpha}}$ is increasing on $\mathbb{N}_{0}$.

The backward difference operator on $h \mathbb{Z}$ is given by $\nabla_{h} \xi(\tau)=\frac{\xi(\tau)-\xi(\tau-h)}{h}$ and the forward operator by $\Delta_{h} \xi(\tau)=\frac{\xi(\tau+h)-\xi(\tau)}{h}$. For $h=1$, we get the backward and forward difference operators $\nabla \xi(\tau)=\xi(\tau)-\xi(\tau-1)$ and $\Delta \xi(\tau)=\xi(\tau+1)-\xi(\tau)$, respectively. The forward jumping operator on the time scale $h \mathbb{Z}$ is $\sigma(\tau)=\tau+h$ and the backward jumping operator is $\rho(\tau)=\tau-h$. For $a, b \in \mathbb{R}$ and $h>0$ we use the notation $\mathbb{N}_{a, h}=$ $\{a, a+h, a+2 h, \ldots\}$ and $b, h \mathbb{N}=\{b, b-h, b-2 h, \ldots\}$.

Definition 2.2. For arbitrary $\tau, \alpha \in \mathbb{R}$ and $h>0$, the nabla $h$-factorial function is defined by

$$
\mathrm{t}_{\mathrm{h}}^{\bar{\alpha}}=\mathrm{h}^{\alpha} \frac{\Gamma\left(\frac{\tau}{\mathrm{h}}+\alpha\right)}{\Gamma\left(\frac{\tau}{h}\right)}
$$

For $h=1$, we write $\tau^{\bar{\alpha}}=\frac{\Gamma(\tau+\alpha)}{\Gamma(\tau)}$.

A straightforward verification leads to

$$
\nabla_{h} \tau_{h}^{\bar{\alpha}}=\alpha \tau_{h}^{\overline{\alpha-1}}
$$

Definition 2.3. [6] For arbitrary $s, \alpha \in \mathbb{R}$ and $h>0$, the delta $h$-factorial function is defined by

$$
s_{h}^{(\alpha)}=h^{\alpha} \frac{\Gamma\left(\frac{s}{h}+1\right)}{\Gamma\left(\frac{s}{h}+1-\alpha\right)} .
$$

For $h=1$, we write $s^{(\alpha)}=\frac{\Gamma(s+1)}{\Gamma(s+1-\alpha)}$. The division by a pole yields to zero.

Notice that

$$
(s+h \alpha-h)_{h}^{(\alpha)}=s_{h}^{\bar{\alpha}} .
$$

The $Q$-operator $(Q f)(t)=f(a+b-t)$ was used in $[7,8]$ to connect left and right fractional sums and differences. In our manuscript, we also use the discrete $\mathrm{Q}$-operator to relate left and right fractional sums and differences and their generalizations to the weighted case.

Definition 2.4. (Nabla $h$-discrete Mittag-Leffler)[9] For $\lambda \in \mathbb{R}$, such that $\left|\lambda h^{\alpha}\right|<1$ and $\alpha, \beta, z \in \mathbb{C}$ with $\operatorname{Re}(\alpha)>0$, the nabla discrete Mittag-Leffler functions is

$$
{ }_{h} \mathrm{E}_{\alpha, \beta}(\lambda, z)=\sum_{k=0}^{\infty} \lambda^{\mathrm{k}} \frac{z_{\mathrm{h}}^{\overline{\mathrm{k \alpha + \beta}-1}}}{\Gamma(\alpha \mathrm{k}+\beta)}, \quad\left|\lambda \mathrm{h}^{\alpha}\right|<1 .
$$


For $\beta=1$, we have

$$
{ }_{h} \mathrm{E}_{\bar{\alpha}}(\lambda, z) \triangleq{ }_{h} \mathrm{E}_{\overline{\alpha, 1}}(\lambda, z)=\sum_{k=0}^{\infty} \lambda^{\mathrm{k}} \frac{z_{\mathrm{h}}^{\overline{\mathrm{k} \alpha}}}{\Gamma(\alpha \mathrm{k}+1)}, \quad\left|\lambda h^{\alpha}\right|<1 .
$$

For more details regarding the Mittag-Leffler functions, we refer to [1, 2].

If we set $\alpha=1$ in (2.6) we recover a representation for the nabla exponential function on $h \mathbb{Z}[16]$.

We shall not give the definition of the delta analogue of Mittag-Leffler function in this article, since we only solve linear equations of nabla type.

\subsection{Left and right delta fractional sums and differences on the isolated time scale}

Definition 2.5. (Delta $h$ - fractional sums) [6] Let $\psi$ be defined on $\mathbb{T}=\mathbb{N}_{a, h} \cap$ b,h $\mathbb{N}$ and assume $b=a+k h$ for some $k \in \mathbb{N}$. Then the left and right delta $h-$ fractional sums of order $\alpha>0$ are given by

$$
\begin{aligned}
\left({ }_{\mathrm{a}} \Delta_{\mathrm{h}}^{-\alpha} \psi\right)(\tau) & =\frac{1}{\Gamma(\alpha)} \int_{\mathrm{a}}^{\sigma(\tau-\alpha h)}(\tau-\sigma(s))_{\mathrm{h}}^{(\alpha-1)} \psi(s) \Delta_{\mathrm{h}} s \\
& =\frac{1}{\Gamma(\alpha)} \sum_{\mathrm{k}=\mathrm{a} / \mathrm{h}}^{\tau / \mathrm{h}-\alpha}(\tau-\sigma(k h))_{\mathrm{h}}^{(\alpha-1)} \psi(k h) h
\end{aligned}
$$

for $\tau \in\{\tau+\alpha h: \tau \in \mathbb{T}\}$ and

$$
\begin{aligned}
\left({ }_{h} \Delta_{b}^{-\alpha} \psi\right)(\tau) & =\frac{1}{\Gamma(\alpha)} \int_{\rho(\tau+\alpha h)}^{b}(\rho(s)-\tau)_{h}^{(\alpha-1)} \psi(s) \nabla_{h} s \\
& =\frac{1}{\Gamma(\alpha)} \sum_{k=\tau / h+\alpha}^{b / h}(k h-\sigma(\tau))_{h}^{(\alpha-1)} \psi(k h) h,
\end{aligned}
$$

for $\tau \in\{\tau-\alpha h: \tau \in \mathbb{T}\}$, respectively, where $b, h \mathbb{N}=\{b, b-h, b-2 h, \ldots\}$.

The next terminology and concepts are borrowed from [9].

Definition 2.6. [9](Delta $h-R L$ fractional differences) Let $\psi$ be defined on $\mathbb{N}_{a, h}$ and $b, h \mathbb{N}$, respectively. Then the left and right delta $h-$ fractional differences of order $\alpha>0$ are defined by

- $\left({ }_{a} \Delta_{h}^{\alpha} \psi\right)(\tau)=\left(\Delta_{h}^{n}{ }_{a} \Delta_{h}^{-(n-\alpha)} \psi\right)(\tau), \quad \tau \in \mathbb{N}_{a+(n-\alpha) h, h}$.

- $\left({ }_{h} \Delta_{b}^{\alpha} \psi\right)(\tau)=\left(\ominus \nabla_{h}^{n} h \Delta_{b}^{-(n-\alpha)} \psi\right)(\tau), \quad \tau \in{ }_{b-(n-\alpha) h, h} \mathbb{N}$,

where $n=[\alpha]+1$. For the order $0<\alpha<1$ compare with Definition 2.8 in [6]. The right fractional difference is here defined different to fit with the dual identities. We shall use the notations $\ominus \nabla_{h}^{n}=(-1)^{n} \nabla_{h}^{n}$ and $\ominus \Delta_{h}^{n}=(-1)^{n} \Delta_{h}^{n}$, where $\nabla_{h}^{n}$ and $\Delta_{h}^{n}$ mean the iteration of the operators $\nabla_{h}$ and $\Delta_{h}$ n-times, respectively. 
Definition 2.7. [9](Delta Caputo $h$-fractional differences) Let $\psi$ be defined on $\mathbb{N}_{a, h}$ and $b, h \mathbb{N}$, respectively. Then the left and right delta Caputo $h-$ fractional differences of order $\alpha>0$ are defined by

- $\left({ }_{\mathrm{a}}^{\mathrm{c}} \Delta_{\mathrm{h}}^{\alpha} \psi\right)(\tau)=\left({ }_{\mathrm{a}} \Delta_{\mathrm{h}}^{-(\mathrm{n}-\alpha)} \Delta_{\mathrm{h}}^{\mathrm{n}} \psi\right)(\tau), \quad \tau \in \mathbb{N}_{\mathrm{a}+(\mathrm{n}-\alpha) \mathrm{h}, \mathrm{h}}$.

- $\left({ }_{h}^{C} \Delta_{b}^{\alpha} \psi\right)(\tau)=\left({ }_{h} \Delta_{b}^{-(n-\alpha)} \ominus \nabla_{h}^{n} \psi\right)(\tau), \quad \tau \in{ }_{b-(n-\alpha) h, h} \mathbb{N}$.

By making use of the $\mathrm{Q}-$ operator we have the following dual identity result which relates left and right $h$-fractional sums and $h$-differences and hence confirm our definitions.

Theorem 2.8. [9] Assume $\alpha>0, \mathrm{~h}>0$ and $\psi$ is defined $\mathbb{N}_{\mathrm{a}, \mathrm{h}}$ in the left case and on $\mathrm{b}, \mathrm{h} \mathbb{N}$ in the right case such that $\mathrm{a}<\mathrm{b}$ and $\mathrm{h}$ divides $\mathrm{b}-\mathrm{a}$. Then,

1. $\left({ }_{\mathrm{a}} \Delta_{\mathrm{h}}^{-\alpha} \mathrm{Q} \psi\right)(\tau)=\mathrm{Q}\left({ }_{\mathrm{h}} \Delta_{\mathrm{b}}^{-\alpha} \psi\right)=\left({ }_{\mathrm{h}} \Delta_{\mathrm{b}}^{-\alpha} \psi\right)(\mathrm{a}+\mathrm{b}-\tau)$.

2. $\left({ }_{\mathrm{a}} \Delta_{\mathrm{h}}^{\alpha} \mathrm{Q} \psi\right)(\tau)=\mathrm{Q}\left({ }_{\mathrm{h}} \Delta_{\mathrm{b}}^{\alpha} \psi\right)(\tau)=\left({ }_{h} \Delta_{\mathrm{b}}^{\alpha} \psi\right)(\mathrm{a}+\mathrm{b}-\tau)$.

3. $\left({ }_{\mathrm{a}}^{\mathrm{c}} \Delta_{\mathrm{h}}^{\alpha} \mathrm{Q} \psi\right)(\tau)=\mathrm{Q}\left({ }_{\mathrm{h}}^{\mathrm{c}} \Delta_{\mathrm{b}}^{\alpha} \psi\right)(\tau)=\left({ }_{\mathrm{h}}^{\mathrm{c}} \Delta_{\mathrm{b}}^{\alpha} \psi\right)(\mathrm{a}+\mathrm{b}-\tau)$.

Lemma 2.9. [9] Let $\alpha>0, \mu>0, \mathrm{~h}>0$. Then,

1.

$$
\mathrm{a}+\mu \mathrm{h} \Delta_{\mathrm{h}}^{-\alpha}(\tau-\mathrm{a})_{\mathrm{h}}^{(\mu-1)}=\frac{\Gamma(\mu)}{\Gamma(\mu+\alpha)}(\tau-a)_{h}^{(\alpha+\mu-1)} .
$$

2.

$$
{ }_{h} \Delta_{b-\mu h}^{-\alpha}(b-\tau)_{h}^{(\mu-1)}=\frac{\Gamma(\mu)}{\Gamma(\mu+\alpha)}(b-\tau)_{h}^{(\alpha+\mu-1)} .
$$

Theorem 2.10. [9] Let $\alpha>0, \mu>0, h>0$. If $\psi$ is defined on $\mathbb{N}_{a, h}$, then for all $\mathrm{t} \in$ $\mathbb{N}_{\mathrm{a}+\mathrm{h}(\alpha+\mu), \mathrm{h}}$, we have

$$
\left({ }_{a}+\alpha h \Delta_{h}^{-\mu}{ }_{a} \Delta_{h}^{-\alpha} \psi\right)(\tau)=\left({ }_{a} \Delta_{h}^{-(\alpha+\mu)} \psi\right)(\tau)=\left({ }_{a}+\mu h \Delta_{h}^{-\alpha}{ }_{a} \Delta_{h}^{-\mu} \psi\right)(\tau) .
$$

Similarly, if $\psi$ is defined on $\mathrm{b}, \mathrm{h} \mathbb{N}$, then for all $\tau \in \mathrm{b}-\mathrm{h}(\alpha+\mu), \mathrm{h} \mathbb{N}$, we have

$$
\left({ }_{h} \Delta_{b-\alpha h}^{-\mu} h \Delta_{b}^{-\alpha} \psi\right)(\tau)=\left({ }_{h} \Delta_{b}^{-(\alpha+\mu)} \psi\right)(\tau)=\left({ }_{h} \Delta_{b-\mu h}^{-\alpha}{ }_{h} \Delta_{b}^{-\mu} \psi\right)(\tau) .
$$

Proposition 2.11. [9] (The relation between delta RL and Caputo $\mathrm{h}$-fractional differences) Assume $\psi$ is defined on $\mathbb{N}_{\mathrm{a}, \mathrm{h}}$ and let $\alpha>0$. Then

$$
\left({ }_{a}^{c} \Delta_{h}^{\alpha} \psi\right)(\tau)=\left({ }_{a} \Delta_{h}^{\alpha} \psi\right)(\tau)-\sum_{k=0}^{n-1} \frac{(t-a)_{h}^{(k-\alpha)}}{\Gamma(k-\alpha+1)} \Delta_{h}^{k} \psi(a), n=[\alpha]+1 .
$$

In particular, for $0<\alpha<1$ we have

$$
\left({ }_{\mathrm{a}}^{\mathrm{C}} \Delta_{\mathrm{h}}^{\alpha} \psi\right)(\tau)=\left({ }_{\mathrm{a}} \Delta_{\mathrm{h}}^{\alpha} \psi\right)(\tau)-\frac{(\tau-\mathrm{a})_{\mathrm{h}}^{(-\alpha)}}{\Gamma(1-\alpha)} \psi(\mathrm{a}) .
$$


On the other hand if $\psi$ is defined on $\mathrm{b}, \mathrm{h} \mathbb{N}$ then

$$
\left({ }_{h}^{c} \Delta_{b}^{\alpha} \psi\right)(\tau)=\left({ }_{h} \Delta_{b}^{\alpha} \psi\right)(\tau)-\sum_{k=0}^{n-1} \frac{(b-\tau)_{h}^{(k-\alpha)}}{\Gamma(k-\alpha+1)} \ominus \nabla_{h}^{k} \psi(b), n=[\alpha]+1 .
$$

In particular, for $0<\alpha<1$ we have

$$
\left({ }_{h}^{C} \Delta_{b}^{\alpha} \psi\right)(\tau)=\left({ }_{h} \Delta_{b}^{\alpha} \psi\right)(\tau)-\frac{(b-\tau)_{h}^{(-\alpha)}}{\Gamma(1-\alpha)} \psi(b) .
$$

Proposition 2.12. [9] For $\alpha>0, h>0$ and $\psi$ defined on $\mathbb{N}_{a, h}$ we have for $t \in \mathbb{N}_{a+n h, h} \subset$ $\mathbb{N}_{a, h}$

$$
\left({ }_{a}+\alpha h \Delta_{h ~}^{\alpha} \Delta_{h}^{-\alpha} \psi\right)(\tau)=\psi(\tau)
$$

and

$$
\begin{gathered}
\left({ }_{a}+(n-\alpha) h-h \Delta_{h}^{-\alpha}{ }_{a} \Delta_{h}^{\alpha} \psi\right)(\tau)=\psi(\tau), \quad \alpha \notin \mathbb{N}, \\
\left({ }_{a} \Delta_{h}^{-\alpha}{ }_{a} \Delta_{h}^{\alpha} \psi\right)(\tau)=\psi(\tau)-\sum_{k=0}^{n-1} \frac{(\tau-a){ }_{h}^{(k)}}{k !} \Delta_{h}^{k} \psi(a), \quad \alpha \in \mathbb{N},
\end{gathered}
$$

where $n=[\alpha]+1$.

Proposition 2.13. [9] For $\alpha>0, h>0$ and $\psi$ defined on $b, h \mathbb{N}$, we have for $\mathrm{t} \in \mathrm{b}-\mathrm{nh}, \mathrm{h} \mathbb{N} \subset$ $b, h \mathbb{N}$

$$
\left({ }_{h} \Delta_{b-\alpha h h}^{\alpha} \Delta_{b}^{-\alpha} \psi\right)(\tau)=\psi(\tau)
$$

and

$$
\begin{gathered}
\left({ }_{h} \Delta_{b-(n-\alpha) h+h}^{-\alpha} \Delta_{b}^{\alpha} \psi\right)(\tau)=\psi(\tau), \quad \alpha \notin \mathbb{N}, \\
\left({ }_{h} \Delta_{b}^{-\alpha}{ }_{h} \Delta_{b}^{\alpha} \psi\right)(\tau)=\psi(\tau)-\sum_{k=0}^{n-1} \frac{(b-\tau)_{h}^{(k)}}{k !} \ominus \nabla_{h}^{k} \psi(b), \quad \alpha \in \mathbb{N} .
\end{gathered}
$$

Lemma 2.14. [9] For any $\alpha>0$ we have

$$
\left({ }_{a} \Delta_{h}^{-\alpha} \Delta_{h} \psi\right)(\tau)=\left(\Delta_{h a} \Delta_{h}^{-\alpha} \psi\right)(t)-\frac{(\tau-a){ }_{h}^{(\alpha-1)}}{\Gamma(\alpha)} \psi(a),
$$

where $\psi$ is defined on $\mathbb{N}_{\mathrm{a}, \mathrm{h}}$. On the other hand, if $\psi$ is defined on $\mathrm{b}, \mathrm{h} \mathbb{N}$, then

$$
\left({ }_{h} \Delta_{b}^{-\alpha}\left(-\nabla_{h}\right) \psi\right)(\tau)=\left(-\nabla_{h}{ }_{h} \Delta_{b}^{-\alpha} \psi\right)(\tau)-\frac{(b-\tau)_{h}^{(\alpha-1)}}{\Gamma(\alpha)} \psi(b) .
$$

Similar to Theorem 4.10 in [18] and by the help of (2.19) and the observation that

$$
\left.{ }_{\mathrm{a}} \Delta_{\mathrm{h}}^{-(1-\alpha)} \psi(\tau)\right|_{\tau=\mathrm{a}+(1-\alpha) \mathrm{h}}=\mathrm{h}^{1-\alpha} \psi(\mathrm{a}),
$$

we can state the following delta discrete fractional Leibniz rule in the sense of RiemannLiouville. 
Lemma 2.15. For $\psi$ defined on $\mathrm{N}_{\mathrm{a}, \mathrm{h}}, \mathrm{h}>0$ and $0<\alpha \leqslant 1$, we have the following identity.

$$
a+(1-\alpha) h \Delta_{h}^{-\alpha}{ }_{a} \Delta_{h}^{\alpha} \psi(\tau)=\psi(\tau)-h^{1-\alpha} \frac{(t-a-(1-\alpha) h)_{h}^{(\alpha-1)} \psi(a)}{\Gamma(\alpha)} .
$$

Proposition 2.16. Assume that $\alpha>0, \mathrm{~h}>0$ and $\psi$ is defined on $\mathbb{N}_{\mathrm{a}, \mathrm{h}}$ and $\mathrm{b}, \mathrm{h}, \mathbb{N}$, respectively. Then

$$
\left(a+(n-\alpha) h \Delta_{h}^{-\alpha} \underset{a}{c} \Delta_{h}^{\alpha} \psi\right)(\tau)=\psi(\tau)-\sum_{k=0}^{n-1} \frac{(\tau-a)_{h}^{(k)}}{k !} \Delta_{h}^{k} \psi(a)
$$

and

$$
\left({ }_{h} \Delta_{b-(n-\alpha) h}^{-\alpha}{ }_{h}^{C} \Delta_{b}^{\alpha} \psi\right)(\tau)=\psi(\tau)-\sum_{k=0}^{n-1} \frac{(b-\tau)_{h}^{(k)}}{k !} \ominus \nabla_{h}^{k} \psi(a) .
$$

\subsection{The nabla fractional sums and differences on the isolated time scale}

In this part, we borrow our notations and their related basics from [9].

Definition 2.17. (Nabla $h$-fractional sums) Let $\rho(\tau)=\tau-h, h>0$ be backward jump operator. Then, for a function $f: \mathbb{N}_{a, h}=\{a, a+h, a+2 h, \ldots\} \rightarrow \mathbb{R}$, the nabla left $h-$ fractional sum of order $\alpha>0$ is given by

$$
\begin{aligned}
\left({ }_{\mathrm{a}} \nabla_{\mathrm{h}}^{-\alpha} \mathrm{f}\right)(\tau) & =\frac{1}{\Gamma(\alpha)} \int_{\mathrm{a}}^{\tau}\left(\tau-\rho_{\mathrm{h}}(\mathrm{s})\right)_{\mathrm{h}}^{\overline{\alpha-1}} \mathrm{f}(\mathrm{s}) \nabla_{\mathrm{h}} \\
& =\frac{1}{\Gamma(\alpha)} \sum_{\mathrm{k}=\mathrm{a} / \mathrm{h}+1}^{\tau / h}\left(\tau-\rho_{\mathrm{h}}(\mathrm{kh})\right)_{\mathrm{h}}^{\overline{\alpha-1}} \mathrm{f}(\mathrm{kh}) \mathrm{h}, \quad \mathrm{t} \in \mathbb{N}_{\mathrm{a}+\mathrm{h}, \mathrm{h} .}
\end{aligned}
$$

The nabla right $h-$ fractional sum of order $\alpha>0$ (ending at $b$ )for $f: b, h \mathbb{N}=\{b, b-$ $h, b-2 h, \ldots\} \rightarrow \mathbb{R}$ is written as

$$
\left({ }_{h} \nabla_{b}^{-\alpha} f\right)(\tau)=\frac{1}{\Gamma(\alpha)} \int_{\tau}^{b}\left(s-\rho_{h}(\tau)\right)_{h}^{\overline{\alpha-1}} f(s) \Delta_{h} s=\frac{1}{\Gamma(\alpha)} \sum_{k=\tau / h}^{b / h-1}\left(k h-\rho_{h}(\tau)\right)_{h}^{\overline{\alpha-1}} f(k h) h .
$$

Definition 2.18. (Nabla $\mathrm{h}-\mathrm{RL}$ fractional differences) The nabla left $\mathrm{h}$-fractional difference of order $\alpha>0$ (starting from $a$ ) has the form

$$
\begin{aligned}
\left({ }_{a} \nabla_{h}^{\alpha} f\right)(\tau) & =\left(\nabla_{h a}^{n} \nabla_{h}^{-(n-\alpha)} f\right)(\tau) \\
& =\frac{\nabla_{h}^{n}}{\Gamma(n-\alpha)} \sum_{k=a / h+1}^{\tau}\left(\tau-\rho_{h}(k h)\right)_{h}^{\overline{n-\alpha-1}} f(k h) h, \quad \tau \in \mathbb{N}_{a+h, h}
\end{aligned}
$$

and the nabla right $h-$ fractional difference of order $\alpha>0$ (ending at $b$ ) is defined as

$$
\begin{aligned}
\left({ }_{h} \nabla_{b}^{\alpha} f\right)(\tau) & =\left(\ominus \Delta_{h h}^{n} \nabla_{b}^{-(n-\alpha)} f\right)(\tau) \\
& =\frac{\ominus \Delta_{h}^{n}}{\Gamma(n-\alpha)} \sum_{k=\tau}^{b / h-1}\left(k h-\rho_{h}(\tau)\right)_{h}^{\overline{n-\alpha-1}} f(k h) h, \quad \tau \in{ }_{b-h, h} \mathbb{N} .
\end{aligned}
$$


Definition 2.19. (The $\mathrm{h}$-Caputo fractional differences) Let $\alpha>0, \mathrm{~h}=[\alpha]+1, \mathrm{~h}>0, \mathrm{a}<$ $b \in \mathbb{R}, a_{h}(\alpha)=a+(n-1) h, b_{h}(\alpha)=b-(n-1) h$. Assume $f$ is defined on $\mathbb{N}_{a, h}=$ $\{a, a+h, a+2 h, \ldots\}$ and on $b, h \mathbb{N}=\{b, b-h, b-2 h, \ldots\}$. We usually have $b=a+k h$ for some $k \in \mathbb{N}$. If $0<\alpha<1$ then $a_{h}(\alpha)=a$ and $b_{h}(\alpha)=b$. Then,

The left $h-$ Caputo fractional difference of order $\alpha$ starting at $a_{h}(\alpha)$ is defined by

$$
\left({ }_{a}^{C} \nabla^{\alpha} f\right)(\tau)=\left({ }_{a_{h}(\alpha)} \nabla^{-(n-\alpha)} \nabla_{h}^{n} f\right)(\tau), \quad \tau \in \mathbb{N}_{a+n h, h} .
$$

The right $h-$ Caputo fractional difference of order $\alpha$ ending at $b_{h}(\alpha)$ is defined by

$$
\left({ }^{C} \nabla_{b}^{\alpha} f\right)(\tau)=\left(\nabla_{b_{h}(\alpha)}^{-(n-\alpha)} \ominus \Delta_{h}^{n} f\right)(\tau), \quad \tau \in b-n h, h \mathbb{N} .
$$

For $h=1$, we obtain the definitions given in $[7,8]$.

Remark 2.20. Alternatively, if in Definition 2.19 we assume that $f$ is defined on $\mathbb{N}_{a-(n-1) h, h}$ for the left case and on $b+(n-1) h, h \mathbb{N}$ for the right case. Then, we define

$$
\left({ }_{a}^{C} \nabla_{h}^{\alpha} f\right)(\tau)={ }_{a} \nabla_{h}^{-(n-\alpha)}\left(\nabla_{h}^{n} f\right)(\tau)
$$

and

$$
\left({ }_{h}^{C} \nabla_{b}^{\alpha} f\right)(\tau)={ }_{h} \nabla_{b}^{-(n-\alpha)}\left(\ominus^{n} \Delta_{h}^{n} f\right)(\tau) .
$$

Lemma 2.21. Let $\operatorname{Re}(\alpha) \geqslant 0, \operatorname{Re}(\mu)>0, \mathrm{~h}>0$. Then,

$$
\begin{aligned}
& { }_{a} \nabla_{h}^{-\alpha}(\tau-a)_{h}^{\bar{\mu}}=\frac{\Gamma(\mu)}{\Gamma(\mu+\alpha)}(\tau-a)_{h}^{\overline{\alpha+\mu-1}}, \operatorname{Re}(\alpha)>0, \\
& { }_{h} \nabla_{b}^{-\alpha}(b-\tau)_{h}^{\bar{\mu}}=\frac{\Gamma(\mu)}{\Gamma(\mu+\alpha)}(b-\tau)_{h}^{\overline{\alpha+\mu-1}}, \operatorname{Re}(\alpha)>0, \\
& { }_{a} \nabla_{h}^{\alpha}(\tau-a)_{h}^{\bar{\mu}}=\frac{\Gamma(\mu)}{\Gamma(\mu-\alpha)}(\tau-a)_{h}^{\overline{\mu-\alpha-1}}, \operatorname{Re}(\alpha) \geqslant 0,
\end{aligned}
$$

and

$$
{ }_{h} \nabla_{b}^{\alpha}(b-\tau)_{h}^{\bar{\mu}}=\frac{\Gamma(\mu)}{\Gamma(\mu-\alpha)}(b-\tau)_{h}^{\overline{\mu-\alpha-1}}, \operatorname{Re}(\alpha) \geqslant 0 .
$$

Lemma 2.22. [9] Let $\alpha>0, \mu>0, h>0$. If $\psi$ is defined on $\mathbb{N}_{a, h}$, then for all $\tau \in \mathbb{N}_{a+h, h}$, we have

$$
\left({ }_{a} \nabla_{h}^{-\mu}{ }_{a} \nabla_{h}^{-\alpha} \psi\right)(\tau)=\left({ }_{a} \nabla_{h}^{-(\alpha+\mu)} \psi\right)(\tau)=\left({ }_{a} \nabla_{h}^{-\alpha}{ }_{a} \nabla_{h}^{-\mu} \psi\right)(\tau) .
$$

Similarly, if $\psi$ is defined on $\mathrm{b}, \mathrm{h} \mathbb{N}$, then for all $\tau \in \mathrm{b}-\mathrm{h}, \mathrm{h} \mathbb{N}$, we have

$$
\left({ }_{h} \nabla_{b}^{-\mu}{ }_{h} \nabla_{b}^{-\alpha} \psi\right)(\tau)=\left({ }_{h} \nabla_{b}^{-(\alpha+\mu)} \psi\right)(\tau)=\left({ }_{h} \nabla_{b}^{-\alpha}{ }_{h} \nabla_{b}^{-\mu} \psi\right)(\tau) .
$$

Lemma 2.23. [9] Assume $\alpha>0, h>0$ and $\psi$ is defined on $\mathbb{N}_{a, h}$ in the left case and on $\mathrm{b}, \mathrm{h} \mathbb{N}$ in the right case such that $\mathrm{a}<\mathrm{b}$ and $\mathrm{h}$ divides $\mathrm{b}-\mathrm{a}$. Then,

- $\left({ }_{a} \nabla_{h}^{-\alpha} Q \psi\right)(\tau)=Q\left({ }_{h} \nabla_{b}^{-\alpha} f\right)(\tau)=\left({ }_{h} \nabla_{b}^{-\alpha} \psi\right)(a+b-\tau)$. 
- $\left({ }_{\mathrm{a}} \nabla_{\mathrm{h}}^{\alpha} \mathrm{Q} \psi\right)(\tau)=\mathrm{Q}\left({ }_{\mathrm{h}} \nabla_{\mathrm{b}}^{\alpha} \psi\right)(\tau)=\left({ }_{\mathrm{h}} \nabla_{\mathrm{b}}^{\alpha} \psi\right)(\mathrm{a}+\mathrm{b}-\tau)$.

- $\left({ }_{\mathrm{a}}^{\mathrm{c}} \nabla_{\mathrm{h}}^{\alpha} \mathrm{Q} \psi\right)(\tau)=\mathrm{Q}\left({ }_{\mathrm{h}}^{\mathrm{c}} \nabla_{\mathrm{b}}^{\alpha} \psi\right)(\tau)=\left({ }_{\mathrm{h}}^{\mathrm{c}} \nabla_{\mathrm{b}}^{\alpha} \psi\right)(\mathrm{a}+\mathrm{b}-\tau)$.

Lemma 2.24. (Nabla Leibniz for left Caputo fractional differences) Assume $\alpha, \mathrm{h}>0$ and $\xi$ is defined on a suitable domain $\mathbb{N}_{\mathrm{a}-(\mathrm{n}-1) \mathrm{h}}, \mathrm{n}=[\alpha]+1$. Then,

$$
\left({ }_{a} \nabla_{h}^{-\alpha} \underset{a}{c} \nabla_{h}^{\alpha} \psi\right)(\tau)=\psi(\tau)-\sum_{k=0}^{n-1} \frac{(\tau-a)_{h}^{\bar{k}}}{k !} \nabla_{h}^{k} \psi(a) .
$$

Alternatively, above we can define on $\mathbb{N}_{a}$ and obtain the following form of Leibniz

$$
\left(a_{h}(\alpha) \nabla_{h}^{-\alpha \underset{a_{h}(\alpha)}{c}} \nabla_{h}^{\alpha} \psi\right)(\tau)=\psi(\tau)-\sum_{k=0}^{n-1} \frac{(\tau-a)_{h}^{\bar{k}}}{k !} \nabla_{h}^{k} \psi(a),
$$

where $a_{h}(\alpha)=a+(n-1) h$.

Lemma 2.25. (Nabla Leibniz for left Caputo fractional differences) Assume $\alpha, \mathrm{h}>0$ and $\psi$ is defined on a suitable domain $\mathbb{N}_{\mathrm{a}-(\mathrm{n}-1) \mathrm{h}}, \mathrm{n}=[\alpha]+1$. Then,

$$
\left({ }_{a} \nabla_{h}^{-\alpha}{ }_{a}^{c} \nabla_{h}^{\alpha} \psi\right)(\tau)=\psi(\tau)-\sum_{k=0}^{n-1} \frac{(t-a)_{h}^{\bar{k}}}{k !} \nabla_{h}^{k} \psi(a) .
$$

Alternatively, above we can define $\psi$ on $\mathbb{N}_{a}$ and obtain the following form of Leibniz

$$
\left(a_{h}(\alpha) \nabla_{h}^{-\alpha} \underset{a_{h}(\alpha)}{c} \nabla_{h}^{\alpha} \psi\right)(\tau)=\psi(\tau)-\sum_{k=0}^{n-1} \frac{\left(t-a_{h}(\alpha)\right)_{h}^{\bar{k}}}{k !} \nabla_{h}^{k} \psi\left(a_{h}(\alpha)\right),
$$

where $a_{h}(\alpha)=a+(n-1) h$.

Lemma 2.26. (Nabla Leibniz for right Caputo fractional differences) Assume $\alpha, \mathrm{h}>0$ and $\psi$ is defined on a suitable domain $\mathrm{b}+(\mathrm{n}-1) \mathrm{h} \mathbb{N}, \mathrm{n}=[\alpha]+1$. Then,

$$
\left({ }_{h} \nabla_{b}^{-\alpha} \underset{h}{c} \nabla_{b}^{\alpha} \psi\right)(\tau)=\psi(\tau)-\sum_{k=0}^{n-1} \frac{(\tau-a)_{h}^{\bar{k}}}{k !} \ominus \Delta_{h}^{k} \psi(b) .
$$

Alternatively, above we can define $\psi$ on $b-(n-1) h \mathbb{N}$ and obtain the following form of Leibniz rule

$$
\left({ }_{b_{h}(\alpha)} \nabla_{h}^{-\alpha} \underset{b_{h}(\alpha)}{c} \nabla_{h}^{\alpha} \psi\right)(\tau)=\psi(\tau)-\sum_{k=0}^{n-1} \frac{\left(b_{h}(\alpha)-t\right)_{h}^{\bar{k}}}{k !} \ominus \Delta_{h}^{k} \psi\left(b_{h}(\alpha)\right),
$$

where $b_{h}(\alpha)=b-(n-1) h$.

If we let $\rho=1$ in Proposition 5.1 of [11] we obtain the following essential tool lemma. 
Lemma 2.27. For any $\alpha \in \mathbb{C}$ with $\operatorname{Re}(\alpha)>0, \mathrm{n}=[\operatorname{Re}(\alpha)]+1$ and $\mathrm{f}$ defined on a suitable domain, we have

$$
\left({ }_{a}^{c} \nabla_{h}^{\alpha} f\right)(\tau)=\left({ }_{a} \nabla_{h}^{\alpha} f\right)(\tau)-\sum_{k=0}^{n-1} \frac{(\tau-a)_{h}^{\overline{k-\alpha}}}{\Gamma(k+1-\alpha)}\left(\nabla_{h}^{k} f\right)(a) .
$$

Similarly, by applying the $\mathrm{Q}-$ operator, we have

$$
\left({ }_{h}^{c} \nabla_{b}^{\alpha} f\right)(\tau)=\left({ }_{h} \nabla_{b}^{\alpha} f\right)(\tau)-\sum_{k=0}^{n-1} \frac{(b-t)_{h}^{\overline{k-\alpha}}}{\Gamma(k+1-\alpha)}\left(\ominus_{\ominus} \Delta_{h}^{k} f\right)(b),
$$

where $\ominus_{\mathrm{h}}^{\mathrm{k}}=(-1)^{\mathrm{k}} \Delta_{\mathrm{h}}^{\mathrm{k}}$.

\section{Weighted left and right fractional sums and differences}

\subsection{In the nabla setting}

Definition 3.1. (Left weighted fractional sums into nabla) The left nabla weighted fractional sum of a function $\eta$ defined on $\mathbb{N}_{a, h}=\{a, a+h, a+2 h, \ldots\}$ via a function $\omega$ defined on $\mathbb{N}_{a, h}$ of order $\alpha, \operatorname{Re}(\alpha)>0$ and starts at $a$, is defined by

$$
\left({ }_{a} \nabla_{\omega, h}^{-\alpha} \eta\right)(t)=\frac{1}{\omega(t)}{ }_{a} \nabla_{h}^{-\alpha}[\omega(t) \eta(t)]=\frac{1}{\omega(t) \Gamma(\alpha)} \int_{a}^{t}\left(t-\rho_{h}(s)\right)_{h}^{\overline{\alpha-1}} \omega(s) \eta(s) \nabla_{h} s .
$$

Definition 3.2. (Left weighted fractional difference into nabla) The left nabla weighted fractional difference of a function $\eta$ defined on $\mathbb{N}_{a, h}=\{a, a+h, a+2 h, \ldots\}$ via a function $\omega$ defined on $\mathbb{N}_{a, h}$ of order $\alpha, \operatorname{Re}(\alpha)>0$ and starts at $a$, is defined by

$$
\left({ }_{a} \nabla_{\omega, h}^{\alpha} \eta\right)(t)=\frac{1}{\omega(t)}{ }_{a} \nabla_{h}^{\alpha}[\omega(t) \eta(t)]=\frac{1}{\omega(t) \Gamma(-\alpha)} \int_{a}^{t}\left(t-\rho_{h}(s)\right)_{h}^{-\alpha-1} \omega(s) \eta(s) \nabla_{h} s .
$$

Next, as usual, we give the definitions in the right case so that it can be verified by the role of summation by parts.

Definition 3.3. (Right weighted fractional sums into nabla) The right nabla weighted fractional sum of a function $\eta$ defined on $b, h \mathbb{N}=\{b, b-h, a-2 h, \ldots\}$ via a function $\omega$ defined on $b, h \mathbb{N}$ of order $\alpha, \operatorname{Re}(\alpha)>0$ and ends at $b$, is defined by

$$
\left(\omega, h \nabla_{b}^{-\alpha} \eta\right)(t)=\omega(t) h \nabla_{b}^{-\alpha}\left[\omega^{-1}(t) \eta(t)\right]=\frac{\omega(t)}{\Gamma(\alpha)} \int_{t}^{b}\left(s-\rho_{h}(t)\right)_{h}^{\overline{\alpha-1}} \omega^{-1}(s) \eta(s) \Delta_{h} s .
$$

Definition 3.4. (Right weighted fractional difference into nabla) The right nabla weighted fractional difference of a function $\eta$ defined on $b, h \mathbb{N}=\{b, b-h, a-2 h, \ldots\}$ via a function $\omega$ defined on $b, h \mathbb{N}$ of order $\alpha, \operatorname{Re}(\alpha)>0$ and ends at $b$, is defined by

$$
\left(\omega, h \nabla_{b}^{\alpha} \eta\right)(t)=\omega(t){ }_{h} \nabla_{b}^{\alpha}\left[\omega^{-1}(t) \eta(t)\right]=\frac{\omega(t)}{\Gamma(-\alpha)} \int_{t}^{b}\left(s-\rho_{h}(t)\right)_{h}^{-\alpha-1} \omega^{-1}(s) \eta(s) \Delta_{h} s .
$$


Proposition 3.5. (Nabla summation by parts) Assume $\eta$ and $\xi$ are defined on $\mathbb{N}_{\mathrm{a}, \mathrm{h}} \cap{ }_{\mathrm{b}, \mathrm{h}} \mathbb{N}$, $\mathrm{b}=\mathrm{a}+\mathrm{kh}$ for some $\mathrm{k} \in \mathbb{N}$. Then, we have

$$
\int_{a}^{b-h} \xi(s)\left(a \nabla_{\omega, h}^{-\alpha} \eta\right)(s) \nabla_{h} s=\int_{a}^{b-h} \eta(s)\left(w, h \nabla_{b}^{-\alpha} \xi\right)(s) \nabla_{h} s .
$$

Proof. From Definition 3.1 we have

$$
\int_{a}^{b-h} \xi(s)\left({ }_{a} \nabla_{\omega, h}^{-\alpha} \eta\right)(s) \nabla_{h} s=\int_{a}^{b-h} \xi(s) \frac{\omega^{-1}(s)}{\Gamma(\alpha)}\left[\int_{a}^{s}\left(s-\rho_{h}(r)\right)_{h}^{\overline{\alpha-1}} \omega(r) \eta(r) \nabla_{h} r\right] \nabla_{h} s .
$$

If we interchange the order of integration or h-summation, we get

$$
\begin{aligned}
& \int_{a}^{b-h} \xi(s)\left(a \nabla_{\omega, h}^{-\alpha} \eta\right)(s) \nabla_{h} s \\
= & \int_{a}^{b-h} \eta(r) \frac{\omega(r)}{\Gamma(\alpha)}\left[\int_{r}^{b-h}\left(s-\rho_{h}(r)\right)_{h}^{\alpha-1} \omega^{-1}(s) \xi(s) \Delta_{h} s\right] \nabla_{h} r \\
= & \int_{a}^{b-h} \eta(r)\left(\omega, h \nabla_{b}^{-\alpha} \xi\right)(r) \nabla_{h} r .
\end{aligned}
$$

Proposition 3.6. If $\left(\nabla_{\omega, h}^{1} \eta\right)(t)=\omega^{-1}(t) \nabla_{h}[\omega(t) \eta(t)]$ and $\left(\nabla_{\omega, h}^{n} \eta\right)(t)$ is the applying of $\nabla_{\omega, \mathrm{h}}^{1}$ on $\eta$ for $\mathrm{n}$ times, then

$$
\left(\mathrm{a} \nabla_{\omega, \mathrm{h}}^{\alpha} \eta\right)(\mathrm{t})=\left(\nabla_{\omega, \mathrm{h}}^{\mathrm{n}} \mathrm{a} \nabla_{\omega, \mathrm{h}}^{-(\mathrm{n}-\alpha)} \eta\right)(\mathrm{t}), \quad \mathrm{n}=[\mathfrak{R}(\alpha)]+1 .
$$

Proof. Using the identities

$$
\nabla_{\mathrm{h}} \int_{\mathrm{a}}^{\mathrm{t}} \Psi(\mathrm{s}, \mathrm{t}) \nabla_{\mathrm{h}} \mathrm{s}=\int_{\mathrm{a}}^{\mathrm{t}} \nabla_{\mathrm{h}} \Psi(\mathrm{s}, \mathrm{t}) \nabla_{\mathrm{h}} \mathrm{s}-\Psi(\mathrm{t}, \mathrm{t}-\mathrm{h}),
$$

$\nabla_{\mathrm{h}}$ with respect to $t$, and

$$
\nabla_{h}\left(t-\rho_{h}(s)\right)_{h}^{\bar{\alpha}}=\alpha\left(t-\rho_{h}(s)\right)_{h}^{\overline{\alpha-1}},
$$

we have for $n=1$

$$
\begin{aligned}
\left(\nabla_{\omega, h}^{1} a \nabla_{\omega, h}^{-(n-\alpha)} \eta\right)(t) & =\omega^{-1}(t) \nabla_{h}\left[\omega(t) \frac{\omega^{-1}(t)}{\Gamma(1-\alpha)} \int_{a}^{t}\left(t-\rho_{h}(s)\right)_{h}^{-\alpha} \eta(s) \omega(s) \nabla_{h} s\right] \\
& =\frac{\omega^{-1}(t)}{\Gamma(-\alpha)} \int_{a}^{t}\left(t-\rho_{h}(s)\right)_{h}^{-\alpha-1} \eta(s) \omega(s) \nabla_{h} s \\
& =\left({ }_{a} \nabla_{\omega, h}^{\alpha} \eta\right)(t) .
\end{aligned}
$$

The rest of the proof follows by induction. 
Proposition 3.7. If $\left(\nabla_{\omega, \mathrm{h}}^{\ominus 1} \eta\right)(\mathrm{t})=-\omega(\mathrm{t}) \Delta_{\mathrm{h}}\left[\omega^{-1}(\mathrm{t}) \eta(\mathrm{t})\right]$ and $\left(\nabla_{\omega, \mathrm{h}}^{\ominus \mathrm{n}} \eta\right)(\mathrm{t})$ is the applying of $\nabla_{\omega, \mathrm{h}}^{\ominus 1}$ on $\eta$ for $\mathrm{n}$ times, then

$$
\left(\omega, \mathrm{h} \nabla_{\mathrm{b}}^{\alpha} \eta\right)(\mathrm{t})=\left(\nabla_{\omega, \mathrm{h}}^{\ominus \mathrm{n}} \omega, \mathrm{h} \nabla_{\mathrm{b}}^{-(\mathrm{n}-\alpha)} \eta\right)(\mathrm{t}), \quad \mathrm{n}=[\mathfrak{R}(\alpha)]+1 .
$$

Proof. The proof is similar to that in Proposition 3.30 and follows by the identities

$$
\Delta_{h} \int_{t}^{b} \Psi(s, t) \Delta_{h} s=\int_{t}^{b} \Delta_{h} \Psi(s, t) \Delta_{h} s-\Psi(t, t+h),
$$

$\Delta_{h}$ with respect to $t$, and

$$
\Delta_{h}\left(s-\rho_{h}(t)\right)_{h}^{\bar{\alpha}}=-\alpha\left(s-\rho_{h}(t)\right)_{h}^{\overline{\alpha-1}} .
$$

In fact, Proposition 3.5 is also valid when we replace $\alpha$ by $-\alpha$. Hence, the following integration by parts is also valid.

Proposition 3.8. (Nabla by parts the weighted fractional differences) Assume $\eta$ and $\xi$ are defined on $\mathbb{N}_{\mathrm{a}, \mathrm{h}} \cap \mathrm{b}, \mathrm{h} \mathbb{N}, \mathrm{b}=\mathrm{a}+\mathrm{kh}$ for some $\mathrm{k} \in \mathbb{N}$. Then, we have

$$
\int_{a}^{b-h} \xi(s)\left(a \nabla_{\omega, h}^{\alpha} \eta\right)(s) \nabla_{h} s=\int_{a}^{b-h} \eta(s)\left(w, h \nabla_{b}^{\alpha} \xi\right)(s) \nabla_{h} s .
$$

Alternatively, we can define them by verifying via the action of the Q-operator which is defined by $Q \eta(t)=\eta(a+b-t)$.

Definition 3.9. (Q-Right weighted fractional sums into nabla) The left nabla weighted fractional sum of a function $\eta$ defined on $b, h \mathbb{N}=\{b, b-h, a-2 h, \ldots\}$ via a function $\omega$ defined on $b, h \mathbb{N}$ of order $\alpha, \operatorname{Re}(\alpha)>0$ and ends $a t b$, is defined by

$$
\begin{aligned}
\left(\underset{\omega, h}{\mathrm{Q}} \nabla_{\mathrm{b}}^{-\alpha} \eta\right)(\mathrm{t}) & =\frac{1}{(\mathrm{Q} \omega)(\mathrm{t})} \mathrm{h} \nabla_{\mathrm{b}}^{-\alpha}[(\mathrm{Q} \omega)(\mathrm{t}) \eta(\mathrm{t})] \\
& =\frac{1}{(\mathrm{Q} \omega)(\mathrm{t}) \Gamma(\alpha)} \int_{\mathrm{t}}^{\mathrm{b}}\left(\mathrm{s}-\rho_{\mathrm{h}}(\mathrm{t})\right)_{\mathrm{h}}^{\overline{\alpha-1}}(\mathrm{Q} \omega)(\mathrm{s}) \eta(\mathrm{s}) \Delta_{\mathrm{h}} \mathrm{s} .
\end{aligned}
$$

Definition 3.10. (Q-Right weighted fractional difference into nabla) The right nabla weighted fractional difference of a function $\eta$ defined on $b, h \mathbb{N}=\{b, b-h, a-2 h, \ldots\}$ via a function $\omega$ defined on $b, h \mathbb{N}$ of order $\alpha, \operatorname{Re}(\alpha)>0$ and ends at $b$, is defined by

$$
\begin{aligned}
\left(\begin{array}{c}
\mathrm{Q}, \mathrm{h} \\
\mathrm{h}
\end{array} \nabla_{\mathrm{b}}^{\alpha} \eta\right)(\mathrm{t}) & =\frac{1}{(\mathrm{Q} \omega)(\mathrm{t})} \mathrm{h} \nabla_{\mathrm{b}}^{\alpha}[(\mathrm{Q} \omega)(\mathrm{t}) \eta(\mathrm{t})] \\
& =\frac{1}{(\mathrm{Q} \omega)(\mathrm{t}) \Gamma(-\alpha)} \int_{\mathrm{t}}^{\mathrm{b}}\left(\mathrm{s}-\rho_{\mathrm{h}}(\mathrm{t})\right)_{\mathrm{h}}^{\overline{-\alpha-1}}(\mathrm{Q} \omega)(\mathrm{s}) \eta(\mathrm{s}) \Delta_{\mathrm{h}} \mathrm{s} .
\end{aligned}
$$

Remark 3.11. The weighted fractional sums and differences have the following properties: 
1. If $\omega$ satisfies $\omega(t) \omega(a+b-t)=1$, the weighted fractional sums and differences defined in by the verification of by parts coincide with the corresponding $\mathrm{Q}$-ones. In particular, it is the case when $\omega(t)=1$, which gives the classical discrete fractional operators. Another example, when $\omega(t)=\frac{t}{b-t}$ and $a=0$. Notice that, this $\omega(t)$ is strictly increasing on $b-h, h \mathbb{N}$.

2. The following $\mathrm{Q}-$ relations are valid:

- $\mathrm{Q}\left({ }_{\mathrm{a}} \nabla_{\omega, \mathrm{h}}^{-\alpha} \eta\right)(\mathrm{t})=\left(\underset{\omega, \mathrm{h}}{\mathrm{Q}} \nabla_{\mathrm{b}}^{-\alpha} \mathrm{Q} \eta\right)(\mathrm{t})$.

- $\mathrm{Q}\left({ }_{\mathrm{a}} \nabla_{\omega, \mathrm{h}}^{\alpha} \eta\right)(\mathrm{t})=\left(\underset{\omega, \mathrm{h}}{\mathrm{Q}} \nabla_{\mathrm{b}}^{\alpha} \mathrm{Q \eta}\right)(\mathrm{t})$.

- $\left(a_{a} \nabla_{\omega, h a}^{-\mu} \nabla_{\omega, h}^{-\alpha} \eta\right)(t)={ }_{a} \nabla_{\omega, h}^{-(\alpha+\mu)} \eta(t)$.

- $\left(\omega, \mathrm{h} \nabla_{\mathrm{b}}^{-\alpha} \omega, \mathrm{h} \nabla_{\mathrm{b}}^{-\mu} \eta\right)(\mathrm{t})=\omega, \mathrm{h} \nabla_{\mathrm{b}}^{-(\alpha+\mu)} \eta(\mathrm{t})$.

- $\left(\underset{\omega, h}{\mathrm{Q}} \nabla_{\mathrm{b}}^{-\alpha} \underset{\omega, \mathrm{h}}{\mathrm{Q}} \nabla_{\mathrm{b}}^{-\mu} \eta\right)(\mathrm{t})=\underset{\omega, \mathrm{h}}{\mathrm{Q}} \nabla_{\mathrm{b}}^{-(\alpha+\mu)} \eta(\mathrm{t})$, provided that

$$
\omega(t) \omega(a+b-t)=1 \text {. }
$$

3. If the weighted function $\omega(t)$ satisfies the following tempered type conditions

(a) $\omega(t+s)=\omega(t) \omega(s)$

(b) $\omega(-t)=\omega^{-1}(t)$ and $\omega^{-1}(-t)=\omega(t)$,

then, we have

- $\left({ }_{a} \nabla_{\omega, h}^{-\alpha} Q \psi\right)(t)=Q\left(\omega, h \nabla_{b}^{-\alpha} f\right)(t)=\left(\omega, h \nabla_{b}^{-\alpha} \psi\right)(a+b-t)$.

- $\left({ }_{a} \nabla_{\omega, h}^{\alpha} \mathrm{Q} \psi\right)(\mathrm{t})=\mathrm{Q}\left(\omega, \mathrm{h} \nabla_{\mathrm{b}}^{\alpha} \mathrm{f}\right)(\mathrm{t})=\left(\omega, \mathrm{h} \nabla_{\mathrm{b}}^{\alpha} \psi\right)(\mathrm{a}+\mathrm{b}-\mathrm{t})$.

- $\left({ }_{a}^{c} \nabla_{\omega, h}^{\alpha} Q \psi\right)(t)=Q\left({ }_{\omega, h}^{c} \nabla_{b}^{\alpha} f\right)(t)=\left(\underset{\omega, h}{c} \nabla_{b}^{\alpha} \psi\right)(a+b-t)$.

The proof can be done by definition and Lemma 2.23. For example, to prove the first part, by the help of the first part of Lemma 2.23 and the properties of $\omega(t)$, we have

$$
\begin{aligned}
\mathrm{Q}\left(\omega, \mathrm{h} \nabla_{\mathrm{b}}^{-\alpha} \psi\right)(\mathrm{t}) & =\mathrm{Q}\left[\omega(\mathrm{t})_{\mathrm{h}} \nabla_{\mathrm{b}}^{-\alpha}\left(\omega^{-1}(\mathrm{t}) \psi(\mathrm{t})\right)\right] \\
& =\omega(\mathrm{a}+\mathrm{b}-\mathrm{t}) \mathrm{a} \nabla_{\mathrm{h}}^{-\alpha}\left(\omega^{-1}(\mathrm{a}+\mathrm{b}-\mathrm{t}) \psi(\mathrm{a}+\mathrm{b}-\mathrm{t})\right) \\
& =\omega(\mathrm{a}) \omega(\mathrm{b}) \omega(-\mathrm{t}) \omega^{-1}(\mathrm{a}) \omega^{-1}(\mathrm{~b}){ }_{\mathrm{a}} \nabla_{\mathrm{h}}^{-\alpha}\left(\omega^{-1}(-\mathrm{t}) \mathrm{Q} \psi(\mathrm{t})\right) \\
& =\omega^{-1}(\mathrm{t}) \mathrm{a} \nabla_{\mathrm{h}}^{-\alpha}\left(\omega^{1}(\mathrm{t}) \mathrm{Q} \psi(\mathrm{t})\right) \\
& =\left(\mathrm{a} \nabla_{\omega, \mathrm{h}}^{-\alpha} \mathrm{Q} \psi\right)(\mathrm{t}) .
\end{aligned}
$$

The proof the other parts is similar. However, in the proof of the second part, we make use of the second part of Lemma 2.23 and the third part by making use of the third part of Lemma 2.23.

Above, we called this weighted by tempered type, since we can choose $\omega(t)$ the nabla exponential function and $\omega^{-1}(t)$ its minus circle exponential function [16].

Theorem 3.12. (The semi-group property for tempered sums) Let $\alpha>0, \mu>0, h>0$. If $\psi$ is defined on $\mathbb{N}_{\mathrm{a}, \mathrm{h}}$, then for all $\mathrm{t} \in \mathbb{N}_{\mathrm{a}+\mathrm{h}, \mathrm{h}}$, we have

$$
\left({ }_{a} \nabla_{\omega, h a}^{-\mu} \nabla_{\omega, h}^{-\alpha} \psi\right)(t)=\left({ }_{a} \nabla_{\omega, h}^{-(\alpha+\mu)} \psi\right)(t)=\left({ }_{a} \nabla_{\omega, h}^{-\alpha}{ }_{a} \nabla_{\omega, h}^{-\mu} \psi\right)(t) .
$$


Similarly, if $\psi$ is defined on $\mathrm{b}, \mathrm{h} \mathbb{N}$, then for all $\mathrm{t} \in \mathrm{b}-\mathrm{h}, \mathrm{h} \mathbb{N}$, we have

$$
\left(\omega, \mathrm{h} \nabla_{\mathrm{b}}^{-\mu} \omega, \mathrm{h} \nabla_{\mathrm{b}}^{-\alpha} \psi\right)(\mathrm{t})=\left(\omega, \mathrm{h} \nabla_{\mathrm{b}}^{-(\alpha+\mu)} \psi\right)(\mathrm{t})=\left(\omega, \mathrm{h} \nabla_{\mathrm{b}}^{-\alpha} \omega, \mathrm{h} \nabla_{\mathrm{b}}^{-\mu} \psi\right)(\mathrm{t}) .
$$

Proof. By the help of Lemma 2.22, we have

$$
\begin{aligned}
\left(a \nabla_{\omega, h}^{-\mu} a \nabla_{\omega, h}^{-\alpha} \psi\right)(t) & =\omega^{-1}(t) a \nabla_{\omega, h}^{-\mu}\left[\omega(t) a \nabla_{\omega, h}^{-\alpha} \psi(t)\right] \\
& =\omega^{-1}(t) a \nabla_{\omega, h}^{-\mu}\left(\omega(t) \omega^{-1}(t)\right. \\
& \left.\times a \nabla_{h}^{-\alpha}[\omega(t) \psi(t)]\right) \\
& =\omega^{-1}(t) a \nabla_{\omega, h}^{-\mu}\left(a \nabla_{h}^{-\alpha}[\omega(t) \psi(t)]\right) \\
& =\omega^{-1}(t) a \nabla_{h}^{-(\alpha+\mu)}[\omega(t) \psi(t)] \\
& =\left(a \nabla_{\omega, h}^{-(\alpha+\mu)} \psi\right)(t) .
\end{aligned}
$$

The proof of (3.21) is similar.

\subsection{In the delta setting}

Definition 3.13. (Left weighted fractional sums into delta) The left delat weighted fractional sum of a function $\eta$ defined on $\mathbb{N}_{a, h}=\{a, a+h, a+2 h, \ldots\}$ via a function $\omega$ defined on $\mathbb{N}_{a, h}$ of order $\alpha, \operatorname{Re}(\alpha)>0$ and starts at $a$, is defined by

$$
\begin{aligned}
\left({ }_{a} \Delta_{\omega, h}^{-\alpha} \eta\right)(t) & =\frac{1}{\omega(t)}{ }_{a} \Delta_{h}^{-\alpha}[\omega(t) \eta(t)] \\
& =\frac{1}{\omega(t) \Gamma(\alpha)} \int_{a}^{\sigma_{h}(t-\alpha h)}\left(t-\sigma_{h}(s)\right)_{h}^{(\alpha-1)} \omega(s) \eta(s) \Delta_{h} s, t \in \mathbb{N}_{a+(3 h, 2, h)}
\end{aligned}
$$

Definition 3.14. (Left weighted fractional difference into delta) The left delta weighted fractional difference of a function $\eta$ defined on $\mathbb{N}_{a, h}=\{a, a+h, a+2 h, \ldots\}$ via a function $\omega$ defined on $\mathbb{N}_{\mathrm{a}, \mathrm{h}}$ of order $\alpha, \operatorname{Re}(\alpha)>0$ and starts at $a$, is defined by

$$
\begin{aligned}
\left({ }_{\mathrm{a}} \Delta_{\omega, \mathrm{h}}^{\alpha} \eta\right)(\mathrm{t}) & =\frac{1}{\omega(\mathrm{t})}{ }_{\mathrm{a}} \Delta_{\mathrm{h}}^{\alpha}[\omega(\mathrm{t}) \eta(\mathrm{t})] \\
& =\frac{1}{\omega(\mathrm{t}) \Gamma(-\alpha)} \int_{\mathrm{a}}^{\sigma_{\mathrm{h}}(\mathrm{t}-\alpha \mathrm{h})}\left(\mathrm{t}-\sigma_{\mathrm{h}}(\mathrm{s})\right)_{\mathrm{h}}^{(-\alpha-1)} \omega(\mathrm{s}) \eta(\mathrm{s}) \Delta_{\mathrm{h}} \mathrm{s},
\end{aligned}
$$

where $t \in \mathbb{N}_{a+(n-\alpha) h, h}$.

Definition 3.15. (Right weighted fractional sums into delta) The right delta weighted fractional sum of a function $\eta$ defined on $b, h \mathbb{N}=\{b, b-h, a-2 h, \ldots\}$ via a function $\omega$ defined on $b, h \mathbb{N}$ of order $\alpha, \operatorname{Re}(\alpha)>0$ and ends at $b$, is defined by

$$
\begin{aligned}
\left(\omega, h \Delta_{b}^{-\alpha} \eta\right)(t) & =\omega(t){ }_{h} \Delta_{b}^{-\alpha}\left[\omega^{-1}(t) \eta(t)\right] \\
& =\frac{\omega(t)}{\Gamma(\alpha)} \int_{\rho_{h}(t+\alpha h)}^{b}\left(s-\sigma_{h}(t)\right)_{h}^{(\alpha-1)} \omega^{-1}(s) \eta(s) \nabla_{h} s,
\end{aligned}
$$

where $t \in b-\alpha h, h \mathbb{N}$. 
Definition 3.16. (Right weighted fractional difference into delta) The right delta weighted fractional difference of a function $\eta$ defined on $b, h \mathbb{N}=\{b, b-h, a-2 h, \ldots\}$ via a function $\omega$ defined on $b, h \mathbb{N}$ of order $\alpha, \operatorname{Re}(\alpha)>0$ and ends at $b$, is defined by

$$
\begin{aligned}
\left(\omega, h \Delta_{b}^{\alpha} \eta\right)(t) & =\omega(t)_{h} \Delta_{b}^{\alpha}\left[\omega^{-1}(t) \eta(t)\right] \\
& =\frac{\omega(t)}{\Gamma(-\alpha)} \int_{\rho_{h}(t+\alpha h)}^{b}\left(s-\sigma_{h}(t)\right)_{h}^{(-\alpha-1)} \omega^{-1}(s) \eta(s) \nabla_{h} s,
\end{aligned}
$$

where $t \in b-(n-\alpha) h, h \mathbb{N}$.

Remark 3.17. For $\omega(t)=t$ in Definition 3.13 and Definition 3.15, we get the definitions in Section 1.3 in [9].

The following fractional summation by parts is a modification of Proposition 4.5 in [8] to $h$-step and under a weight function.

Proposition 3.18. (Delta summation by parts) Assume $\eta$ and $\xi$ are defined on $\mathbb{N}_{\mathrm{a}, \mathrm{h}} \cap \mathrm{b}, \mathrm{h}$, $\mathrm{b}=\mathrm{a}+\mathrm{kh}$ for some $\mathrm{k} \in \mathbb{N}$. Then, we have

$$
\int_{a+h}^{b} \xi(s)\left(a+h \Delta_{\omega, h}^{-\alpha} \eta\right)(s+\alpha h) \Delta_{h} s=\int_{a+h}^{b} \eta(s)\left(\omega, h \Delta_{b-h}^{-\alpha} \xi\right)(s-\alpha h) \Delta_{h} s .
$$

Proof. The proof is similar to that in Proposition 3.5 and follows by definition, interchanging the order of h-summations and by noting that $s-\sigma_{h}(r-\alpha h)=s+\alpha h-$ $\sigma_{h}(r)$.

Proposition 3.19. If $\left(\Delta_{\omega, h}^{1} \eta\right)(t)=\omega^{-1}(t) \Delta_{h}[\omega(t) \eta(t)]$ and $\left(\Delta_{\omega, h}^{n} \eta\right)(t)$ is the applying of $\Delta_{\omega, h}^{1}$ on $\eta$ for $n$ times, then

$$
\left(\mathrm{a}_{\mathrm{a}, \mathrm{h}}^{\alpha} \eta\right)(\mathrm{t})=\left(\Delta_{\omega, \mathrm{h}}^{\mathrm{n}} \mathrm{a} \Delta_{\omega, \mathrm{h}}^{-(\mathrm{n}-\alpha)} \eta\right)(\mathrm{t}), \quad \mathrm{n}=[\mathfrak{R}(\alpha)]+1 .
$$

Proof. The proof is similar to that in Proposition 3.7. In fact, it follows by employing the identities Using the identities

$$
\Delta_{h} \int_{a}^{t+\alpha h} \Psi(s, t) \Delta_{h} s=\int_{a}^{t+\alpha h+h} \Delta_{h} \Psi(s, t) \Delta_{h} s+\Psi(t+\alpha h, t+h), t \in \mathbb{N}_{a+(1-\alpha) h, h}
$$

$\nabla_{\mathrm{h}}$ with respect to $\mathrm{t}$, and

$$
\Delta_{h}\left(t-\sigma_{h}(s)\right)_{h}^{(\alpha)}=\alpha\left(t-\sigma_{h}(s)\right)_{h}^{(\alpha-1)} .
$$

Proposition 3.20. If $\left(\Delta_{\omega, \mathrm{h}}^{\ominus 1} \eta\right)(\mathrm{t})=-\omega(\mathrm{t}) \nabla_{\mathrm{h}}\left[\omega^{-1}(\mathrm{t}) \eta(\mathrm{t})\right]$ and $\left(\Delta_{\omega, \mathrm{h}}^{\ominus \mathrm{n}} \eta\right)(\mathrm{t})$ is the applying of $\Delta_{\omega, \mathrm{h}}^{\ominus 1}$ on $\eta$ for $\mathrm{n}$ times, then

$$
\left(\omega, \mathrm{h} \Delta_{\mathrm{b}}^{\alpha} \eta\right)(\mathrm{t})=\left(\Delta_{\omega, \mathrm{h}}^{\ominus n} \omega, \mathrm{h} \Delta_{\mathrm{b}}^{-(\mathrm{n}-\alpha)} \eta\right)(\mathrm{t}), \quad \mathrm{n}=[\Re(\alpha)]+1
$$


Proof. The proof is similar to that in Proposition 3.19 and follows by the identities

$$
\nabla_{\mathrm{h}} \int_{\mathrm{t}-\alpha \mathrm{h}}^{\mathrm{b}} \Psi(\mathrm{s}, \mathrm{t}) \nabla_{\mathrm{h}} \mathrm{s}=\int_{\mathrm{t}-\alpha \mathrm{h}}^{\mathrm{b}} \nabla_{\mathrm{h}} \Psi(\mathrm{s}, \mathrm{t}) \nabla_{\mathrm{h}} \mathrm{s}-\Psi(\mathrm{t}-\alpha \mathrm{h}, \mathrm{t}),
$$

$\nabla_{h}$ with respect to $t$, and

$$
\nabla_{h}\left(s-\sigma_{h}(t)\right)_{h}^{(\alpha)}=-\alpha\left(s-\sigma_{h}(t)\right)_{h}^{(\alpha-1)} .
$$

In fact, Proposition 3.18 is also valid when we replace $\alpha$ by $-\alpha$. Hence, the following integration by parts is also valid.

Proposition 3.21. (Delta by parts of the weighted fractional differences) Assume $\eta$ and $\xi$ are defined on $\mathbb{N}_{\mathrm{a}, \mathrm{h}} \cap \mathrm{b}, \mathrm{h} \mathbb{N}, \mathrm{b}=\mathrm{a}+\mathrm{kh}$ for some $\mathrm{k} \in \mathbb{N}$. Then, we have

$$
\int_{a+h}^{b} \xi(s)\left(a+h \Delta_{\omega, h}^{\alpha} \eta\right)(s-\alpha h) \Delta_{h} s=\int_{a+h}^{b} \eta(s)\left(w, h \Delta_{b-h}^{\alpha} \xi\right)(s+\alpha h) \Delta_{h} s .
$$

Remark 3.22. The same steps can be carried to the delta case as done in Definition 3.9, Definition 3.10 and in Remark 3.11. However, nabla will be replaced by delta and the action of the Q-operator for delta fractional sums and differences will be used. Also, we have to care about the domain of the delta defined fractional difference and sum operators.

\section{The weighted Caputo fractional differences and sums}

\subsection{In the nabla setting}

Definition 4.1. (The weighted $h-C a p u t o$ fractional differences) Let $\alpha>0, n=[\alpha]+1, h>$ $0, a<b \in \mathbb{R}$, and assume $f$ and $\omega$ are defined on $\mathbb{N}_{a-(n-1) h, h}$ for the left case and on $b+(n-1) h, h \mathbb{N}$ for the right case. Then the left weighted $h$-Caputo fractional difference of order $\alpha$ starting at $a$ and via $\omega$ is defined by

$$
\left({ }_{a}^{C} \nabla_{\omega, h}^{\alpha} f\right)(t)={ }_{a} \nabla_{\omega, h}^{-(n-\alpha)}\left(\nabla_{\omega, h}^{n} f\right)(t),
$$

and the right one ending at $b$ by

$$
\left(\underset{\omega, h}{C} \nabla_{b}^{\alpha} f\right)(t)={ }_{\omega, h} \nabla_{b}^{-(n-\alpha)}\left(\nabla_{\omega, h}^{\ominus n} f\right)(t),
$$

where $\left(\nabla_{\omega, h}^{n} \eta(t)\right)$ is the application of $\left(\nabla_{\omega, h}^{1} \eta\right)(t)=\omega^{-1}(t) \nabla_{h}[\omega(t) \eta(t)] n-$ times, and $\left(\nabla_{\omega, h}^{\ominus n} \eta\right)(t)$ is the application of $\left(\nabla_{\omega, h}^{\ominus 1} \eta\right)(t)=-\omega(t) \Delta_{h}\left[\omega^{-1}(t) \eta(t)\right], n-$ times.

For $h=1, \omega(t)=1$, we obtain the definitions given in $[7,8]$. 
Remark 4.2. Alternatively, if in Definition 4.1 we assume that $f$ is defined on $\mathbb{N}_{a-(n-1) h, h}$ for the left case and on $b+(n-1) h, h \mathbb{N}$ for the right case, the weighted left and right $h$ Caputo differences via $\omega$ are defined by

$$
\left({ }_{a}^{C} \nabla_{\omega, h}^{\alpha} f\right)(t)=\omega^{-1}(t){ }_{a}^{C} \nabla_{h}^{\alpha}[\omega(t) f(t)], \quad t \in \mathbb{N}_{a-h, h} .
$$

The right $\omega$-weighted $h$-Caputo fractional difference of order $\alpha$ ending at $b$ is defined by

$$
\left(\underset{\omega, h}{C} \nabla_{b}^{\alpha} f\right)(t)=\omega(t){ }_{h}^{C} \nabla_{b}^{\alpha}\left[\omega^{-1}(t) f(t)\right], \quad t \in b-h, h \mathbb{N} .
$$

This clear by splitting $\left(\nabla_{\omega, h}^{n} \eta(t)\right)$ in the left case and $\left(\nabla_{\ominus \omega, h}^{n} \eta(t)\right)$ in the right case.

Theorem 4.3. (The relation between weighted Riemann and Caputo types) For any $\alpha \in \mathbb{C}$, with $\operatorname{Re}(\alpha)>0, \mathrm{n}=[\operatorname{Re}(\alpha)]+1$ and $\xi, \omega$ defined on a suitable domain, we have

$$
\begin{aligned}
\left({ }_{a}^{C} \nabla_{\omega, h}^{\alpha} \xi\right)(t) & =\left(a \nabla_{\omega, h}^{\alpha} \xi\right)(t)-\omega^{-1}(t) \\
& \times \sum_{k=0}^{n-1} \frac{(t-a) \bar{h}}{\Gamma(k+1-\alpha)}\left(\nabla_{h}^{k}[\omega(t) \xi(t)]\right)(a) .
\end{aligned}
$$

Similarly, for the right case, we have

$$
\begin{aligned}
\left(\underset{\omega, h}{\mathrm{C}} \nabla_{\mathrm{b}}^{\alpha} \xi\right)(\mathrm{t}) & =\left(\omega, \mathrm{h} \nabla_{\mathrm{b}}^{\alpha} \xi\right)(\mathrm{t}) \\
& -\omega(\mathrm{t}) \sum_{\mathrm{k}=0}^{\mathrm{n}-1} \frac{(\mathrm{b}-\mathrm{t})_{\mathrm{h}}^{\overline{k-\alpha}}}{\Gamma(\mathrm{k}+1-\alpha)}\left(\ominus^{\mathrm{k}} \Delta_{\mathrm{h}}^{\mathrm{k}}\left[\omega^{-1}(\mathrm{t}) \xi(\mathrm{t})\right]\right)(\mathrm{b}) .
\end{aligned}
$$

Proof. In (2.38) set $f(t)=\omega(t) \xi(t)$ to get

$$
\begin{aligned}
\left({ }_{\mathrm{a}}^{\mathrm{C}} \nabla_{\mathrm{h}}^{\alpha} \omega(\mathrm{t}) \xi(\mathrm{t})\right)(\mathrm{t}) & =\left({ }_{\mathrm{a}} \nabla_{\mathrm{h}}^{\alpha} \omega(\mathrm{t}) \xi(\mathrm{t})\right)(\mathrm{t}) \\
& -\sum_{\mathrm{k}=0}^{\mathrm{n}-1} \frac{(\mathrm{t}-\mathrm{a})_{\mathrm{h}}^{\bar{k}-\alpha}}{\Gamma(k+1-\alpha)}\left(\nabla_{h}^{\mathrm{k}}[\omega(\mathrm{t}) \xi(\mathrm{t})]\right)(\mathrm{a}) .
\end{aligned}
$$

The multiplication of (4.5) by $\omega^{-1}(\mathrm{t})$ will imply that

$$
\begin{aligned}
\left({ }_{a}^{C} \nabla_{\omega, h}^{\alpha} \xi(t)\right)(t) & =\left({ }_{a} \nabla_{\omega, h}^{\alpha} \xi\right)(t) \\
& -\omega^{-1}(t) \sum_{k=0}^{n-1} \frac{(t-a)_{h}^{\overline{k-\alpha}}}{\Gamma(k+1-\alpha)}\left(\nabla_{h}^{k}[\omega(t) \xi(t)]\right)
\end{aligned}
$$

To prove the right case set $f(t)=\omega^{-1}(t) \xi(t)$ in (2.39) and then multiply by $\omega(t)$. 


\subsection{In the delta setting}

Definition 4.4. (Left weighted Caputo fractional difference into delta) The left delta weighted fractional difference of a function $\eta$ defined on $\mathbb{N}_{a, h}=\{a, a+h, a+2 h, \ldots\}$ via a function $\omega$ defined on $\mathbb{N}_{a, h}$ of order $\alpha, \operatorname{Re}(\alpha)>0$ and starts at $a$, is defined by

$$
\left({ }_{a}^{c} \Delta_{\omega, h}^{\alpha} \eta\right)(t)=\frac{1}{\omega(t)}{ }_{a}^{c} \Delta_{h}^{\alpha}[\omega(t) \eta(t)],
$$

where $t \in \mathbb{N}_{a+(n-\alpha) h, h}$ and ${ }_{a}^{C} \Delta_{h}^{\alpha}$ is the delta Caputo fractional difference.

Definition 4.5. (Right Caputo weighted fractional differences into delta) The right delta weighted fractional sum of a function $\eta$ defined on $b, h \mathbb{N}=\{b, b-h, a-2 h, \ldots\}$ via $a$ function $\omega$ defined on $b, h \mathbb{N}$ of order $\alpha, \operatorname{Re}(\alpha)>0$ and ends at $b$, is defined by

$$
\left(\underset{\omega, h}{C} \Delta_{b}^{\alpha} \eta\right)(t)=\omega(t){ }_{h}^{C} \Delta_{b}^{\alpha}\left[\omega^{-1}(t) \eta(t)\right],
$$

where $t \in b-(n-\alpha) h, h \mathbb{N}$ and ${ }_{h}^{C} \Delta_{b}^{\alpha}$ is the right Caputo fractional difference.

Theorem 4.6. (The relation between weighted Riemann and Caputo types) For any $\alpha \in \mathbb{C}$ with $\operatorname{Re}(\alpha)>0, \mathrm{n}=[\operatorname{Re}(\alpha)]+1$ and $\xi, \omega$ defined on a suitable domain, we have

$$
\begin{aligned}
\left({ }_{\mathrm{a}}^{\mathrm{C}} \Delta_{\omega, \mathrm{h}}^{\alpha} \xi\right)(\mathrm{t}) & =\left({ }_{a} \Delta_{\omega, h}^{\alpha} \xi\right)(\mathrm{t})-\omega^{-1}(\mathrm{t}) \\
& \times \sum_{k=0}^{n-1} \frac{(\mathrm{t}-\mathrm{a})_{h}^{(k-\alpha)}}{\Gamma(k+1-\alpha)}\left(\Delta_{h}^{\mathrm{k}}[\omega(\mathrm{t}) \xi(\mathrm{t})]\right)(\mathrm{a}) .
\end{aligned}
$$

Similarly, for the right case, we have

$$
\begin{aligned}
\left(\underset{\omega, h}{\mathrm{C}} \Delta_{\mathrm{b}}^{\alpha} \xi\right)(\mathrm{t}) & =\left(\omega, \mathrm{h} \Delta_{\mathrm{b}}^{\alpha} \xi\right)(\mathrm{t}) \\
& -\omega(\mathrm{t}) \sum_{k=0}^{\mathrm{n}-1} \frac{(\mathrm{b}-\mathrm{t})_{\mathrm{h}}^{(\mathrm{k}-\alpha)}}{\Gamma(\mathrm{k}+1-\alpha)}\left(\ominus \nabla_{\mathrm{h}}^{\mathrm{k}}\left[\omega^{-1}(\mathrm{t}) \xi(\mathrm{t})\right]\right)(\mathrm{b}) .
\end{aligned}
$$

Proof. In Proposition 2.11 set $f(t)=\omega(t) \xi(t)$ to get

$$
\begin{aligned}
\left({ }_{\mathrm{a}}^{\mathrm{C}} \Delta_{\mathrm{h}}^{\alpha} \omega(\mathrm{t}) \xi(\mathrm{t})\right)(\mathrm{t}) & =\left({ }_{\mathrm{a}} \Delta_{h}^{\alpha} \omega(\mathrm{t}) \xi(\mathrm{t})\right)(\mathrm{t}) \\
& \left.-\sum_{\mathrm{k}=0}^{\mathrm{n}-1} \frac{(\mathrm{t}-\mathrm{a})_{\mathrm{h}}^{\mathrm{k}-\alpha}}{\Gamma(k+1-\alpha)}\left(\Delta_{h}^{\mathrm{k}}[\omega(\mathrm{t})) \xi(\mathrm{t})\right]\right)(\mathrm{a}) .
\end{aligned}
$$

The multiplication of (4.11) by $\omega^{-1}(\mathrm{t})$ will imply that

$$
\begin{aligned}
\left({ }_{\mathrm{a}}^{\mathrm{C}} \Delta_{\omega, \mathrm{h}}^{\alpha} \xi(\mathrm{t})\right)(\mathrm{t}) & =\left({ }_{\mathrm{a}} \Delta_{\omega, \mathrm{h}}^{\alpha} \xi\right)(\mathrm{t}) \\
& -\omega^{-1}(\mathrm{t}) \sum_{\mathrm{k}=0}^{\mathrm{n}-1} \frac{(\mathrm{t}-\mathrm{a})_{\mathrm{h}}^{(\mathrm{k}-\alpha)}}{\Gamma(k+1-\alpha)}\left(\Delta_{h}^{k}[\omega(\mathrm{t}) \xi(\mathrm{t})]\right)
\end{aligned}
$$

To prove the right case set $f(t)=\omega^{-1}(t) \xi(t)$ in the right identity in Proposition 2.11 and then multiply by $\omega(t)$. 


\section{The Leibniz type rules}

This section provides the tool to transform fractional for nabla and delta weighted difference equations to summation equations so that the method of successive approximation can be applied.

The first theorem is dealing with the Caputo case in the nabla setting.

Theorem 5.1. (The application of the sum on the Caputo weighted difference- Leibniz Rule)

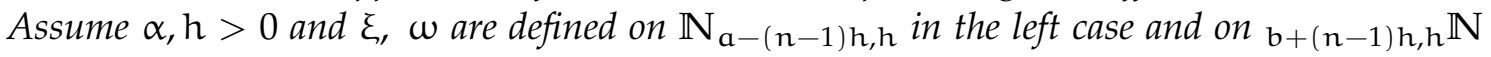
in the right case. Then, we have

$$
{ }_{a} \nabla_{\omega, h}^{-\alpha} \underset{a}{c} \nabla_{\omega, h}^{\alpha} \xi(t)=\left[\xi(t)-\omega^{-1}(t) \sum_{k=0}^{n-1} \frac{(t-a)_{h}^{\bar{k}}}{k !}\left(\nabla_{h}^{k} \omega(t) \xi(t)\right)(a)\right],
$$

and

$$
\omega, h \nabla_{b}^{-\alpha} \underset{\omega, h}{c} \nabla_{b}^{\alpha} \xi(t)=\left[\xi(t)-\omega(t) \sum_{k=0}^{n-1} \frac{(b-t)_{h}^{\bar{k}}}{k !}\left(\left(\ominus_{\ominus} \Delta_{h}^{k} \omega^{-1}(t) \xi(t)\right)\right)(b)\right] .
$$

In particular, if $0<\alpha \leqslant 1$, we have

$$
{ }_{a} \nabla_{\omega, h}^{-\alpha} \underset{a}{c} \nabla_{\omega, h}^{\alpha} \xi(t)=\xi(t)-\omega^{-1}(t) \omega(a) \xi(a),
$$

and

$$
\omega, \mathrm{h} \nabla_{\mathrm{b}}^{-\alpha} \omega, \mathrm{h} \nabla_{\mathrm{b}}^{\alpha} \xi(\mathrm{t})=\xi(\mathrm{t})-\omega(\mathrm{t}) \omega^{-1}(\mathrm{~b}) \xi(\mathrm{b}) .
$$

Proof. Using the $\omega$-weighted fractional sum and Caputo difference definitions and by applying the Leibniz rule (2.34), we obtain

$$
\begin{aligned}
{ }_{a} \nabla_{\omega, h}^{-\alpha}{ }_{a}^{C} \nabla_{\omega, h}^{\alpha} \xi(t) & =\omega^{-1}(t){ }_{a} \nabla_{h}^{-\alpha}\left[\omega(t){ }_{a}^{c} \nabla_{\omega, h}^{\alpha} \xi(t)\right] \\
& =\omega^{-1}(t){ }_{a} \nabla_{h}^{-\alpha}\left[\omega(t) \omega^{-1}(t){ }_{a}^{C} \nabla_{h}^{\alpha}(\omega(t) \xi(t))\right] \\
& =\omega^{-1}(t){ }_{a} \nabla_{h}^{-\alpha}{ }_{a}^{C} \nabla_{h}^{\alpha}[\omega(t) \xi(t)] \\
& =\omega^{-1}(t)\left[\omega(t) \xi(t)-\sum_{k=0}^{n-1} \frac{(t-a)_{h}^{\bar{k}}}{k !}\left(\nabla_{h}^{k} \omega(t) \xi(t)\right)(a)\right] \\
& =\left[\xi(t)-\omega^{-1}(t) \sum_{k=0}^{n-1} \frac{(t-a)_{h}^{\bar{k}}}{k !}\left(\nabla_{h}^{k} \omega(t) \xi(t)\right)(a)\right]
\end{aligned}
$$

The right case is similar and can be proved by making use of (2.36).

Remark 5.2. Using the definitions and the fact that ${ }_{a} \nabla_{h}^{-\alpha}{ }_{a} \nabla_{h}^{\alpha} \xi(t)=\xi(t)$, it is straightforward to show that

$$
{ }_{a} \nabla_{\omega, h \quad a}^{-\alpha} \nabla_{\omega, h}^{\alpha} \xi(t)=\xi(t) .
$$

Indeed, we have 


$$
\begin{aligned}
{ }_{a} \nabla_{\omega, h}^{-\alpha} a \nabla_{\omega, h}^{\alpha} \xi(t) & =\omega^{-1}(t){ }_{a} \nabla_{h}^{-\alpha}\left[\omega(t)\left({ }_{a} \nabla_{h}^{\alpha} \xi\right)(t)\right] \\
& =\omega^{-1}(t) a \nabla_{h}^{-\alpha}\left[\omega(t) \omega^{-1}(t)\left(a{ }_{a} \nabla_{h}^{\alpha} \omega(t) \xi\right)(t)\right] \\
& =\omega^{-1}(t) \omega(t) \xi(t) \\
& =\xi(t) .
\end{aligned}
$$

Hence, it logical to generate fractional $\omega$-weighted initial value difference equations in the setting of Riemann by starting at $a-1$. For example the following system is of interest

$$
a-h \nabla_{\omega, h}^{\alpha} \xi(t)=f(t, x(t)), \quad \xi(a)=c_{0}, 0<\alpha \leqslant 1, h>0 .
$$

For the $h=1, \omega(t)=1$ case we refer to [8]. For the $h \mathbb{Z}$ case with $\omega(t)=1$ we refer to [12]. Indeed, in [12], for $\alpha \in(0,1]$, it was proved that

$$
{ }_{a} \nabla_{h}^{-\alpha}{ }_{a-h} \nabla_{h}^{\alpha} \xi(t)=\xi(t)-\frac{h^{1-\alpha}}{\Gamma(\alpha)}(t-a+h)_{h}^{\overline{\alpha-1}} \xi(a) .
$$

As a result of (5.7), it is straight froward to show that

$$
{ }_{a} \nabla_{\omega, h a-h}^{-\alpha} \nabla_{\omega, h}^{\alpha} \xi(t)=\xi(t)-\frac{h^{1-\alpha}}{\Gamma(\alpha)}(t-a+h)_{h}^{\overline{\alpha-1}} \omega^{-1}(t) \omega(a) \xi(a) .
$$

The next theorem is about the delta Caputo weighted Leibniz rule whose proof is similar to the nabla case and can be proved by the help of the delta Caputo Leibniz rule in the non-weighted case as stated in Proposition 2.16.

Theorem 5.3. (The application of the sum on the Caputo weighted difference- Leibniz Rule) Assume $\alpha, \mathrm{h}>0$ and $\xi, \omega$ are defined on $\mathbb{N}_{\mathrm{a}, \mathrm{h}}$ in the left case and on $\mathrm{b}, \mathrm{h} \mathbb{N}$ in the right case. Then, we have

$$
a+(n-\alpha) h \Delta_{\omega, h}^{-\alpha} \underset{a}{c} \Delta_{\omega, h}^{\alpha} \xi(t)=\left[\xi(t)-\omega^{-1}(t) \sum_{k=0}^{n-1} \frac{(t-a)_{h}^{(k)}}{k !}\left(\Delta_{h}^{k} \omega(t) \xi(t)\right)(a)\right],
$$

and

$$
\omega, h \Delta_{b-(n-\alpha) h}^{-\alpha} \underset{\omega, h}{\stackrel{c}{c}} \Delta_{b}^{\alpha} \xi(t)=\left[\xi(t)-\omega(t) \sum_{k=0}^{n-1} \frac{(b-t)_{h}^{(k)}}{k !}\left(\left(\ominus \nabla_{h}^{k} \omega^{-1}(t) \xi(t)\right)\right)(b)\right] .
$$

In particular, if $0<\alpha \leqslant 1$, we have

$$
\mathrm{a}+(\mathrm{n}-\alpha) \mathrm{h} \nabla_{\omega, \mathrm{h}}^{-\alpha} \mathrm{c}_{\mathrm{c}}^{\mathrm{C}} \nabla_{\omega, \mathrm{h}}^{\alpha} \xi(\mathrm{t})=\xi(\mathrm{t})-\omega^{-1}(\mathrm{t}) \omega(\mathrm{a}) \xi(\mathrm{a}),
$$

and

$$
\omega, h \nabla_{b-(n-\alpha) h}^{-\alpha} \stackrel{\underset{\omega}{\omega}, h}{\stackrel{\alpha}{\alpha}} \nabla_{b}^{\alpha} \xi(t)=\xi(t)-\omega(t) \omega^{-1}(b) \xi(b) .
$$


Making use of Lemma 2.15, we can state the following Leinbniz rule in the frame of Riemann-Lioulle.

Proposition 5.4. For $\alpha \in(0,1], h>0$ and $\xi$ define on $\mathbb{N}_{\mathrm{a}, \mathrm{h}}$, we have

$$
\mathrm{a}+(1-\alpha) \mathrm{h} \Delta_{\omega, \mathrm{h} a}^{-\alpha} \Delta_{\omega, \mathrm{h}}^{\alpha} \xi(\mathrm{t})=\psi(\mathrm{t})-\mathrm{h}^{1-\alpha} \frac{(\mathrm{t}-\mathrm{a}-(1-\alpha) \mathrm{h})_{\mathrm{h}}^{(\alpha-1)} \omega(\mathrm{a}) \omega^{-1}(\mathrm{t}) \xi(\mathrm{a})}{\Gamma(\alpha)} .
$$

In particular, if $\omega(\mathrm{t})$ is of tempered type, then we have

$$
\mathrm{a}+(1-\alpha) \mathrm{h} \Delta_{\omega, \mathrm{h} a}^{-\alpha} \Delta_{\omega, h}^{\alpha} \xi(\mathrm{t})=\psi(\mathrm{t})-\mathrm{h}^{1-\alpha} \frac{(\mathrm{t}-\mathrm{a}-(1-\alpha) \mathrm{h})_{\mathrm{h}}^{(\alpha-1)} \omega^{-1}(\mathrm{t}-\mathrm{a}) \xi(\mathrm{a})}{\Gamma(\alpha)}
$$

\section{More integration by parts}

\subsection{In the nabla setting}

The following h-discrete tempered integration by parts are to interchange between the Caputo and Riemann differences. In fact, by use the nabla integration by parts on the $h \mathbb{Z}$ time scale [16]:

$$
\int_{a}^{b} f(t) \nabla_{h} g(t) \nabla_{h} t=\left.f(t) g(t)\right|_{a} ^{b}-\int_{a}^{b} g(t-h) \nabla_{h} f(t) \nabla_{h} t, b-a=k h, k \in \mathbb{N} .
$$

Proposition 6.1. (Nabla by parts for the tempered fractional differences from left Caputo to right Riemann ) Assume $\eta$ and $\xi$ are defined on $\mathbb{N}_{\mathrm{a}, \mathrm{h}} \cap \mathrm{b}, \mathrm{h} \mathbb{N}, \mathrm{b}=\mathrm{a}+\mathrm{kh}$ for some $\mathrm{k} \in \mathbb{N}$. Then, we have

$$
\begin{aligned}
\int_{a}^{b-h} \xi(s)\left({ }_{a}^{c} \nabla_{\omega, h}^{\alpha} \eta\right)(s) \nabla_{h} s & =\eta(s) \omega,\left.h \nabla_{b}^{-(1-\alpha)} \xi(s)\right|_{a} ^{b-h} \\
& +\int_{a-h}^{b-2 h} \eta(s) \omega, h \nabla_{b}^{\alpha} \xi(s)(s) \nabla_{s} h
\end{aligned}
$$

Proof. From Remark 4.2, Proposition 3.5 with $\omega(t)=1,(6.1)$, and Definition 3.4 , we proceed by 


$$
\begin{aligned}
& \int_{a}^{b-h} \xi(s)\left({ }_{a}^{c} \nabla_{\omega, h}^{\alpha} \eta\right)(s) \nabla_{h} s \\
= & \int_{a}^{b-h} \xi(s) \omega^{-1}(s){ }_{a}^{c} \nabla_{h}^{\alpha}(\omega(s) \eta(s)) \nabla_{h} s \\
= & \int_{a}^{b-h} \xi(s) \omega^{-1}(s){ }_{a} \nabla_{h}^{-(1-\alpha)} \nabla_{h}[\omega(s) \eta(s)] \nabla_{h} s \\
= & \int_{a}^{b-h} h \nabla_{b}^{-(1-\alpha)}\left[\omega^{-1}(s) \xi(s)\right] \nabla_{h}[\omega(s) \eta(s)] \nabla_{h} s \\
= & \left.\eta(s) \omega(s) \nabla_{b}^{-(1-\alpha)}\left[\omega^{-1}(s) \xi(s)\right]\right|_{a} ^{b-h} \\
- & \int_{a}^{b-h} \eta(s-h) \omega(s-h) \nabla_{h} h \nabla_{b}^{-(1-\alpha)}\left[\omega^{-1}(s) \xi(s)\right] \nabla_{h} s \\
= & \eta(s) \omega,\left.h \nabla_{b}^{-(1-\alpha)} \xi(s)\right|_{a} ^{b-h} \\
+ & \int_{a}^{b-h} \eta(s-h) \omega(s-h)\left(-\Delta_{h}\right){ }_{h} \nabla_{b}^{-(1-\alpha)}\left[\omega^{-1}(s) \xi(s)\right](s-h) \nabla_{h} s \\
= & \eta(s) \omega,\left.h \nabla_{b}^{-(1-\alpha)} \xi(s)\right|_{a} ^{b-h} \\
+ & \int_{a}^{b-h} \eta(s-h) \omega(s-h){ }_{h} \nabla_{b}^{\alpha}\left[\omega^{-1}(s) \xi(s)\right](s-h) \nabla_{h} s \\
= & \eta(s) \omega,\left.h \nabla_{b}^{-(1-\alpha)} \xi(s)\right|_{a} ^{b-h} \\
+ & \int_{a-h}^{b-2 h} \eta(s){ }_{a}^{\alpha} \xi(s)(s) \nabla_{s} h
\end{aligned}
$$

Remark 6.2. If we let $\omega(t)=1$ and $h=1$ in Proposition 6.1, then we recover Theorem 43 in [7].

In the next result, we use the following version of integration by parts [16]:

$$
\int_{a}^{b} f^{\rho}(t) \nabla_{h} g(t) \nabla_{h} t=\left.f(t) g(t)\right|_{a} ^{b}-\int_{a}^{b} g(t) \nabla_{h} f(t) \nabla_{h} t, b-a=k h, k \in \mathbb{N},
$$

where $f^{\rho}(t)=f(t-h)$.

Proposition 6.3. (Nabla by parts for the tempered fractional differences from left Reimann to right Caputo ) Assume $\eta$ and $\xi$ are defined on $\mathbb{N}_{\mathrm{a}, \mathrm{h}} \cap{ }_{\mathrm{b}, \mathrm{h}} \mathbb{N}, \mathrm{b}=\mathrm{a}+\mathrm{kh}$ for some $\mathrm{k} \in \mathbb{N}$. Then, we have

$$
\begin{aligned}
& \int_{a}^{b-h} \xi(s)\left(a \nabla_{\omega, h}^{\alpha} \eta\right)(s) \nabla_{h} s \\
= & \left.\omega^{-1}(s+h){ }_{a} \nabla_{h}^{-(1-\alpha)}[\eta(s) \omega(s)] \xi(s+h)\right|_{a} ^{b-h} \\
+ & \int_{a}^{b-h} \eta(s) \underset{\omega, h}{c} \nabla_{b}^{\alpha} \xi(s) \nabla_{s} h .
\end{aligned}
$$


Proof. From Definition 3.2, (6.5), Proposition 3.5 with $\omega(t)=1$, and Remark 4.2, we proceed by

$$
\begin{aligned}
& \int_{a}^{b-h} \xi(s)\left({ }_{a} \nabla_{w, h}^{\alpha} \eta\right)(s) \nabla_{h} s \\
= & \int_{a}^{b-h} \xi(s) \omega^{-1}(s){ }_{a} \nabla_{h}^{\alpha}(\omega(s) \eta(s)) \nabla_{h} s \\
= & \int_{a}^{b-h}\left(\xi^{\sigma}\right)^{\rho}(s)\left(\left(\omega^{-1}\right)^{\sigma}\right)^{\rho} \nabla_{h} a \nabla_{h}^{-(1-\alpha)}[\omega(s) \eta(s)] \nabla_{h} s \\
= & \left.\omega^{-1}(s+h){ }_{a} \nabla_{h}^{-(1-\alpha)}[\eta(s) \omega(s)] \xi(s+h)\right|_{a} ^{b-h} \\
- & \int_{a}^{b-h} a \nabla_{h}^{-(1-\alpha)}[\omega(s) \eta(s)] \nabla_{h}\left[\xi^{\sigma}(s)\left(\omega^{-1}\right)^{\sigma}(s)\right] \nabla_{s} h \\
= & \left.\omega^{-1}(s+h){ }_{a} \nabla_{h}^{-(1-\alpha)}[\eta(s) \omega(s)] \xi(s+h)\right|_{a} ^{b-h} \\
- & \int_{a}^{b-h} a \nabla_{h}^{-(1-\alpha)}[\omega(s) \eta(s)] \Delta_{h}\left[\xi(s) \omega^{-1}(s)\right] \nabla_{s} h \\
= & \left.\omega^{-1}(s+h){ }_{a} \nabla_{h}^{-(1-\alpha)}[\eta(s) \omega(s)] \xi(s+h)\right|_{a} ^{b-h} \\
+ & \int_{a}^{b-h} \eta(s) \omega(s){ }_{h} \nabla_{b}^{-(1-\alpha)}\left(-\Delta_{h}\right)\left[\xi(s) \omega^{-1}(s)\right] \nabla_{s} h \\
= & \left.\omega^{-1}(s+h){ }_{a} \nabla_{h}^{-(1-\alpha)}[\eta(s) \omega(s)] \xi(s+h)\right|_{a} ^{b-h} \\
+ & \int_{a}^{b-h} \eta(s) \omega(s) \underset{h}{c} \nabla_{b}^{\alpha}\left[\xi(s) \omega^{-1}(s)\right] \nabla_{s} h \\
= & \left.\omega^{-1}(s+h) \underset{a}{a} \nabla_{h}^{-(1-\alpha)}[\eta(s) \omega(s)] \xi(s+h)\right|_{a} ^{b-h} \\
+ & \int_{a}^{b-h} \eta(s) \underset{\omega, h}{c} \nabla_{b}^{\alpha} \xi(s) \nabla_{s} h
\end{aligned}
$$

Remark 6.4. Other integration by parts can be formulated by changing the rule between $\omega$-weighted left and right and Rieman and Caputo settings. Also, all including the above ones can be generalized for the higher order.

\subsection{In the delta setting}

The following h-discrete tempered integration by parts are to interchange between the Caputo and Riemann differences. In fact, by use the nabla integration by parts on the $h \mathbb{Z}$ time scale [15]:

$$
\int_{a}^{b} f(t) \Delta_{h} g(t) \Delta_{h} t=\left.f(t) g(t)\right|_{a} ^{b}-\int_{a}^{b} g(t+h) \Delta_{h} f(t) \Delta_{h} t, b-a=k h, k \in \mathbb{N} .
$$


Proposition 6.5. (Nabla by parts for the tempered fractional differences from left Caputo to right Riemann ) Assume $\eta$ and $\xi$ are defined on $\mathbb{N}_{\mathrm{a}, \mathrm{h}} \cap \mathrm{b}, \mathrm{h} \mathbb{N}, \mathrm{b}=\mathrm{a}+\mathrm{kh}$ for some $\mathrm{k} \in \mathbb{N}$. Then, we have

$$
\begin{aligned}
& \int_{a+h}^{b} \xi(s)\left(\begin{array}{c}
c \\
a+h
\end{array} \Delta_{w, h}^{\alpha} \eta\right)(s+\alpha h) \Delta_{h} s \\
= & \left.\eta(s) \omega(s)\left({ }_{h} \Delta_{b-h}^{-(1-\alpha)}\left[\omega^{-1}(.+\alpha h) \xi(.)\right]\right)(s-\alpha h)\right|_{a} ^{b-h} \\
+ & \int_{a+2 h}^{b+h} \eta(s)\left(\omega(s+\alpha h), h \Delta_{b-h}^{\alpha} \xi\right)(s-\alpha h) \Delta_{h} s .
\end{aligned}
$$

Proof. From Definition 4.4, Proposition 3.18 with $\omega(t)=1$, (6.7), and Definition 3.16 , we proceed by

$$
\begin{aligned}
& \int_{a+h}^{b} \xi(s)\left(\begin{array}{l}
c \\
a+h
\end{array} \Delta_{w, h}^{\alpha} \eta\right)(s+\alpha h) \Delta_{h} s \\
= & \int_{a+h}^{b} \xi(s) \omega^{-1}(s+\alpha h){ }_{a+h}^{c} \Delta_{h}^{\alpha}[\omega(.) \eta(.)](s+\alpha h) \Delta_{h} s \\
= & \int_{a+h}^{b} \xi(s) \omega^{-1}(s+\alpha h)\left({ }_{a+h} \Delta_{h}^{-(1-\alpha)} \Delta_{h}[\omega(.) \eta(.)]\right)(s+\alpha h) \Delta_{h} s \\
= & \int_{a+h}^{b}\left(h \Delta_{b-h}^{-(1-\alpha)}\left[\omega^{-1}(.+\alpha h) \xi(.)\right]\right)(s-\alpha h) \Delta_{h}[\omega(s) \eta(s)] \Delta_{h} s \\
= & \left.\eta(s) \omega(s)\left({ }_{h} \Delta_{b-h}^{-(1-\alpha)}\left[\omega^{-1}(.+\alpha h) \xi(.)\right]\right)(s-\alpha h)\right|_{a} ^{b-h} \\
- & \int_{a+h}^{b} \eta(s+h) \omega(s+h) \Delta_{h}\left({ }_{h} \Delta_{b-h}^{-(1-\alpha)}\left[\omega^{-1}(.+\alpha h) \xi(.)\right]\right)(s-\alpha h) \Delta_{h} s \\
= & \left.\eta(s) \omega(s)\left({ }_{h} \Delta_{b-h}^{-(1-\alpha)}\left[\omega^{-1}(.+\alpha h) \xi(.)\right]\right)(s-\alpha h)\right|_{a} ^{b-h} \\
- & \int_{a+h}^{b} \eta(s+h) \omega(s+h) \nabla_{h}\left({ }_{h} \Delta_{b-h}^{-(1-\alpha)}\left[\omega^{-1}(.+\alpha h) \xi(.)\right]\right)(s+(1-\alpha) h) \Delta_{h} s \\
= & \left.\eta(s) \omega(s)\left({ }_{h} \Delta_{b-h}^{-(1-\alpha)}\left[\omega^{-1}(.+\alpha h) \xi(.)\right]\right)(s-\alpha h)\right|_{a} ^{b-h} \\
+ & \int_{a+h}^{b} \eta(s+h) \omega(s+h)\left({ }_{h} \Delta_{b-h}^{\alpha}\left[\omega^{-1}(.+\alpha h) \xi(.)\right]\right)(s+(1-\alpha) h) \Delta_{h} s \\
= & \left.\eta(s) \omega(s)\left({ }_{h} \Delta_{b-h}^{-(1-\alpha)}\left[\omega^{-1}(.+\alpha h) \xi(.)\right]\right)(s-\alpha h)\right|_{a} ^{b-h} \\
+ & \int_{a+h}^{b} \eta(s+h)\left(\omega(s+\alpha h), h \Delta_{b-h}^{\alpha} \xi\right)(s+(1-\alpha) h) \Delta_{h} s \\
= & \left.\eta(s) \omega(s)\left({ }_{h} \Delta_{b-h}^{-(1-\alpha)}\left[\omega^{-1}(.+\alpha h) \xi(.)\right]\right)(s-\alpha h)\right|_{a} ^{b-h} \\
+ & \int_{a+2 h}^{b+h} \eta(s)\left(w(s+\alpha h), h \Delta_{b-h}^{\alpha} \xi\right)(s-\alpha h) \Delta_{h} s \\
&
\end{aligned}
$$


Remark 6.6. If we let $\omega(t)=1$ and $h=1$ in Proposition 6.5, then we recover the delta version of Theorem 43 in [7].

In the next result, we use the following version of integration by parts [15]:

$$
\int_{a}^{b} f^{\sigma}(t) \nabla_{h} g(t) \Delta_{h} t=\left.f(t) g(t)\right|_{a} ^{b}-\int_{a}^{b} g(t) \Delta_{h} f(t) \Delta_{h} t, b-a=k h, k \in \mathbb{N},
$$

where $f^{\sigma}(t)=f(t+h)$.

Proposition 6.7. (Nabla by parts for the tempered fractional differences from left Reimann to right Caputo ) Assume $\eta$ and $\xi$ are defined on $\mathbb{N}_{\mathrm{a}, \mathrm{h}} \cap \mathrm{b}, \mathrm{h}, \mathrm{N}, \mathrm{b}=\mathrm{a}+\mathrm{kh}$ for some $\mathrm{k} \in \mathbb{N}$. Then, we have

$$
\begin{aligned}
& \int_{a+h}^{b} \xi(s)\left(\underset{a+h}{ } \Delta_{\omega(s-\alpha h), h}^{\alpha} \eta\right)(s+\alpha h) \Delta_{h} s \\
= & \left.\xi(s-h) \omega^{-1}(s-h)\left(a+h \Delta_{h}^{-(1-\alpha)}[\omega(.-\alpha h) \eta(.)]\right)(s+\alpha h)\right|_{a+h} ^{b} \\
+ & \int_{a+h}^{b} \eta(s)\left({ }_{w, h}^{c} \Delta_{b-h}^{\alpha} \xi(s)\right)(s-\alpha h) \Delta_{h} s .
\end{aligned}
$$

Proof. From Definition 3.14, (6.8), Proposition 3.18 with $\omega(t)=1$, and Definition 4.5, we proceed by

$$
\begin{aligned}
& \int_{a+h}^{b} \xi(s)\left(a+h \Delta_{w(s-\alpha h), h}^{\alpha} \eta\right)(s+\alpha h) \Delta_{h} s \\
& =\int_{a+h}^{b} \xi(s) \omega^{-1}(s) a+h \Delta_{h}^{\alpha}[\omega(.-\alpha h) \eta(.)](s+\alpha h) \Delta_{h} s \\
& =\int_{a+h}^{b}(\xi)^{\rho \sigma}(s)\left(\omega^{-1}\right)^{\rho \sigma}(s)\left(\Delta_{h a+h} \Delta_{h}^{-(1-\alpha)}[\omega(.-\alpha h) \eta(.)]\right)(s+\alpha h) \Delta_{h} s \\
& =\left.\xi(s-h) \omega^{-1}(s-h)\left(a+h \Delta_{h}^{-(1-\alpha)}[\omega(.-\alpha h) \eta(.)]\right)(s+\alpha h)\right|_{a+h} ^{b} \\
& -\int_{a+h}^{b} \nabla_{h}\left[\xi(s) \omega^{-1}(s)\right]\left({ }_{a+h} \Delta_{h}^{-(1-\alpha)}[\omega(.-\alpha h) \eta(.)]\right)(s+\alpha h) \Delta_{h} s \\
& =\left.\xi(s-h) \omega^{-1}(s-h)\left(a+h \Delta_{h}^{-(1-\alpha)}[\omega(.-\alpha h) \eta(.)]\right)(s+\alpha h)\right|_{a+h} ^{b} \\
& +\int_{a+h}^{b}\left({ }_{h}^{c} \Delta_{b-h}^{\alpha}\left[\xi(s) \omega^{-1}(s)\right]\right)(s-\alpha h) \omega(s-\alpha h) \eta(s) \Delta_{h} s \\
& =\left.\xi(s-h) \omega^{-1}(s-h)\left(a+h \Delta_{h}^{-(1-\alpha)}[\omega(.-\alpha h) \eta(.)]\right)(s+\alpha h)\right|_{a+h} ^{b} \\
& +\int_{a+h}^{b} \eta(s)\left(\underset{\omega, h}{c} \Delta_{b-h}^{\alpha} \xi(s)\right)(s-\alpha h) \Delta_{h} s
\end{aligned}
$$

Above we have used the observations that $\Delta_{h} g(s+\alpha h)=\left(\Delta_{h} g\right)(s+\alpha h)$ and $\Delta_{h} g^{\rho}(s)=$ $\nabla_{h} g(s)$. 
Remark 6.8. Other integration by parts can be formulated by changing the rule between $\omega-$ weighted left and right and Rieman and Caputo settings. Also, all including the above ones can be generalized for the higher order.

\section{Some examples and solutions linear equations}

Example 7.1. Let $\alpha, \beta \in \mathbb{C}$ such that $\operatorname{Re}(\alpha) \geqslant 0$ and $\operatorname{Re}(\beta)>0$. Then, we have

1. $\left({ }_{a} \nabla_{\omega, h}^{-\alpha} \omega^{-1}(t)(t-a)_{h}^{\overline{\beta-1}}\right)(\tau)=\frac{\Gamma(\beta)}{\Gamma(\beta+\alpha)} \omega^{-1}(\tau)(\tau-a)_{h}^{\overline{\alpha+\beta-1}}, \quad \operatorname{Re}(\alpha)>0$.

2. $\left(\omega, \mathrm{h} \nabla_{\mathrm{b}}^{-\alpha} \omega(\mathrm{t})(\mathrm{b}-\mathrm{t})_{\mathrm{h}}^{\overline{\beta-1}}\right)(\tau)=\frac{\Gamma(\beta)}{\Gamma(\beta+\alpha)} \omega(\tau)(\mathrm{b}-\tau)_{\mathrm{h}}^{\overline{\alpha+\beta-1}}, \quad \operatorname{Re}(\alpha)>0$.

3. $\left.\left({ }_{a} \nabla_{\omega, h}^{\alpha} \omega^{-1}(t)(t-a){ }_{h}^{\overline{\beta-1}}\right)(\tau)=\frac{\Gamma(\beta)}{\Gamma(\beta-\alpha)} \omega^{-1}(\tau)(\tau-a)\right)_{h}^{\overline{\beta-1-\alpha}}, \quad \operatorname{Re}(\alpha) \geqslant 0$.

4. $\left(\omega, \mathrm{h} \nabla_{\mathrm{b}}^{\alpha} \omega(\mathrm{t})(\mathrm{b}-\mathrm{t})_{\mathrm{h}}^{\overline{\beta-1}}\right)(\tau)=\frac{\Gamma(\beta)}{\Gamma(\beta-\alpha)} \omega(\tau)(\mathrm{b}-\tau)_{\mathrm{h}}^{\overline{\beta-1-\alpha}}, \quad \operatorname{Re}(\alpha) \geqslant 0$.

Proof. The proofs follow by definitions and Lemma 2.21.

Example 7.2. Let $\alpha, \beta \in \mathbb{C}$ be such that $\operatorname{Re}(\alpha)>0$ and $\operatorname{Re}(\beta)>0$. Then, for any weighted function $\omega$ is defined on $\mathbb{N}_{a-(n-1) h, h}$ for the left case and on $b+(n-1) h, h \mathbb{N}$ for the right case and $n=[\operatorname{Re}(\alpha)]+1$ we have

1. $\left({ }_{\mathrm{a}}^{\mathrm{C}} \nabla_{\omega, \mathrm{h}}^{\alpha} \omega^{-1}(\mathrm{t})(\mathrm{t}-\mathrm{a})_{\mathrm{h}}^{\overline{\beta-1}}\right)(\tau)=\frac{\Gamma(\beta)}{\Gamma(\beta-\alpha)} \omega^{-1}(\tau)(\tau-\mathrm{a})_{\mathrm{h}}^{\overline{\beta-1-\alpha}}, \quad \operatorname{Re}(\beta)>\mathrm{n}$.

2. $\left({ }_{\omega, h}^{C} \nabla_{b}^{\alpha} \omega(t)(b-t){ }_{h}^{\overline{\beta-1}}\right)(\tau)=\frac{\Gamma(\beta)}{\Gamma(\beta-\alpha)} \omega(\tau)(b-\tau)_{h}^{\overline{\beta-1-\alpha}}, \quad \operatorname{Re}(\beta)>n$.

For $k=0,1, \ldots, n-1$, we have $\left({ }_{a}^{C} \nabla_{\omega, h}^{\alpha} \omega^{-1}(t)(t-a){ }_{h}^{\bar{k}}\right)(\tau)=0$ and $\left(\underset{\omega, h}{C} \nabla_{b}^{\alpha} \omega(t)(b-\right.$ $\left.\mathrm{t})_{\mathrm{h}}^{\bar{k}}\right)(\tau)=0$. In particular, $\left({ }_{\mathrm{a}}^{\mathrm{C}} \nabla_{\mathrm{w}, \mathrm{h}}^{\alpha} \omega^{-1}(\mathrm{t})\right)(\tau)=0$ and $\left(\underset{\omega, \mathrm{h}}{\mathrm{C}} \nabla_{\mathrm{b}}^{\alpha} \omega(\mathrm{t})\right)(\tau)=0$.

Proof. The proofs follow by definitions and Lemma 2.21.

Remark 7.3. From Example 1 in [9], after correcting the typo mistake by changing summation $\sum_{s=a+1}^{t}$ there in (53-55) by $\int_{a}^{t} \nabla_{h} s$ (see the definition of the h-nabla fractional sum in Definition 10 there), recall that the solution of the system

$$
{ }_{a}^{C} \nabla_{h}^{\alpha} u(t)=r u(t)+g(t), \quad u(a)=u_{a}, 0<\alpha \leqslant 1,
$$

has the solution

$$
u(t)=u_{0 h} E_{\bar{\alpha}}(r, t-a)+\int_{a}^{t}{ }_{h} E_{\overline{\alpha, \alpha}}(r, t-s+h) g(s) \nabla_{h} s .
$$

Example 7.4. Consider the linear Caputo discrete $\omega$ - weighted $h$-fractional initial value problem

$$
\left({ }_{a} \nabla_{\omega, h}^{\alpha} \xi\right)(\tau)-r \xi(\tau)=f(\tau), \xi(a)=c_{0}, r, 0<\alpha \leqslant 1 .
$$

Then, $\xi(t)$ is a solution of (7.2) if and only if it satisfies the $h$-integral equation 


$$
\begin{aligned}
\xi(\tau) & =c_{0} \omega^{-1}(\tau){ }_{h} E_{\bar{\alpha}}(r, \tau-a)+\omega^{-1}(\tau) \\
& \times \int_{a}^{\tau}{ }_{h} E_{\overline{\alpha, \alpha}}(r, \tau-s+h) \omega(s) f(s) \nabla_{h} s .
\end{aligned}
$$

In fact, system (7.2) is equivalent to the system

$$
{ }_{a}^{C} \nabla_{h}^{\alpha} u(\tau)=r u(\tau)+\omega(\tau) f(\tau), u(\tau)=\omega(\tau) \xi(\tau), u(a)=c_{0} \omega(a) .
$$

By Remark 7.3, we conclude that

$$
u(\tau)=u(a){ }_{h} E_{\bar{\alpha}}(r, \tau-a)+\int_{a}^{\tau}{ }_{h} E_{\overline{\alpha, \alpha}}(r, \tau-s+h) \omega(s) f(s) \nabla_{h} s .
$$

Then, substituting $\mathfrak{u}(\tau)=w(\tau) \xi(\tau), \mathfrak{u}(a)=c_{0} \omega(a)$ in (7.5) will imply the solution representation (7.3).

If $\omega(t)$ is of discrete exponential type or the equation (7.2) is of tempered type, then the solution representation in (7.3) will have the form

$$
\begin{aligned}
\xi(\tau) & =c_{0} \omega^{-1}(\tau) h E_{\bar{\alpha}}(r, \tau-a)+ \\
& \times \int_{a}^{\tau}{ }_{h} E_{\overline{\alpha, \alpha}}(r, \tau-s+h) \omega^{-1}(\tau-s) f(s) \nabla_{h} s .
\end{aligned}
$$

Alternatively, this example can be solved by the successive approximation method via the Leinbniz rule (5.3) and by making use of the semigroup property (3.20).

\section{Conclusions}

In this work we have accomplished the following:

- To make our generalization easy and understandable for the readers, we have recalled some basics about the delta and nabla discrete fractional calculus on the isolated time scale with step $h \in(0,1]$.

- We have proposed the left and right weighted fractional sums and differences with a study of the action of the Q-operator. In the right case Q-versions of weighted fractional sums and differences and some conditions on the weight $\omega(t)$ have been given to have coincidence with those verifying the integration by parts rule.

- The left and right weighted Caputo fractional differences have been defined and related to the Riemann-Liouville ones into nabla and delta.

- In order to provide a weighted fractional summation transformation method to solve the Caputo difference type equations, we proposed the Liblniez rule facilitating the action of the weighted fractional sum, either left or right, on the weighted Caputo fractional difference. 
- A shifted nabla Leinbniz type rule has been formulated to solve a weighted fractional difference initial value problem within Riemann allowing the appearance of an initial condition.

- Some examples are given in the case of nabla weighted and a weighted Caputo fractional equation has been solved using a change of variable allowing the use of the non-weighted case. The solutions are then expressed by means of the generalized discrete Mittag-Leffler function designed for the insolated time scales with step $h$.

- By the use of the weighted summation by parts we have proved integration by parts formulas in the senses nabla and delta. such by parts formulas allow the pass from the left weight Caputo to weighted right Riemann-Liouville and the pass from left weighted Riemann-Liouville to the right weighted Caputo. In the delta case we observed that the dependency of the domain of the fractional sum and difference operator on the order $\alpha$ is reflected on the dependency of the weight function $\omega$ on the order as well.

- The particular case $\omega(t)=1$ throughout the weighted study results in the classical discrete fractional calculus into nabla and delta which have been studied by many authors in literature. some other particular cases of the weight function $\omega$ may result into interesting discrete fractional operators and of special interest the use of the discrete exponential type functions which discretesizes the tempered calculus. Such special case, due the nice symmetric properties of the discrete delta and nabla exponential functions and their minus circle analogues, deserves to be studied separately.

\section{References}

[1] Kilbas AA, Srivastava HM, Trujillo JJ (2006). "Theory and Application of Fractional Differential Equations". North Holland Mathematics Studies, 204.

[2] Podlubny I (1999). "Fractional Differential Equations". Academic Press, San Diego CA.

[3] Jarad F, Abdeljawad T, Shah K (2020). On the Weighted Fractional Operators of a Function with Respect to Another Function. Fractals 28(8):2040011 https:/ / doi.org/10.1142/S0218348X20400113

[4] Goodrich C, Peterson A (2015). "Discrete Fractional Calculus". Springer.

[5] Atıc1 FM, Eloe PW (2009). Discrete fractional calculus with the nabla operator. Electr. J. Qualit. Theor. Differ. Equ. 2009(3):1-12.

[6] Bastos NRO, Ferreira RAC, Torres DFM (2011). Discrete-time fractional variational problems. Signal Processing 91(3):513-524. https:/ / doi.org/10.1016/j.sigpro.2010.05.001

[7] Abdeljawad T (2013). On delta and nabla Caputo fractional differences and dual identities. Discr. Dynam. Nat. Soc. 2013 Article ID 406910: 12 pages.

[8] Abdeljawad T (2013). Dual identities in fractional difference calculus within Riemann. Adv. Differ. Equ. 2013:36. https:/ / doi.org/10.1186/1687-1847-2013-36

[9] Abdeljawad T (2018). Different type kernel $\mathrm{h}$-fractional differences and their fractional $\mathrm{h}-$ sums. Chaos Solitons Fractals 116: 146-156. https://doi.org/10.1016/j.chaos.2018.09.022

[10] Mozyrska D, Girejko E (2013). "Overview of Fractional h-difference Operators, Operator Theory: Advances and Applications book series". Springer Basel, 229.

[11] Abdeljawad T, Jarad F, Alzabut J (2017). Fractional proportional differences with memory. The European Physical Journal Special Topics, 226(16-18): 3333-3354 (2017).

[12] Suwan I, Owies S, Abdeljawad T (2018). Monotonicity results for h-discrete fractional operators and application. Adv. Differ. Equ. 2018:207. https://doi.org/10.1186/s13662-018-1660-5 
[13] Wu GC, Baleanu D (2014). Discrete fractional logistic map and its chaos. Nonlinear Dyn. 75: $283-287$. https:/ / doi.org/10.1007/s11071-013-1065-7

[14] Wu GC, Baleanu D, Zeng SD (2014). Discrete chaos in fractional sine and standard maps. Phys. Lett. A 378: 484-487.

[15] Bohner M, Peterson A (2001). "Dynamic Equations on Time Scales". Birkhäuser.

[16] Bohner M, Peterson A (2003). "Advances in Dynamic Equations on Time Scales". Birkhäuser.

[17] Cao J, Li C, Chen Y (2014). "On tempered and substantial fractional calculus", in: 2014 IEEE/ASME 10th International Conference on Mechatronic and Embedded Systems and Applications (MESA), IEEE, pp. 1-6.

[18] Atici FM, Uyanik M (2015). Analysis of discrete fractional operators. Appl. Anal. Discrete Math. 9:139-149. https://doi.org/10.2298/AADM150218007A 